\title{
Identification of Flap endonuclease 1 as a potential core gene in hepatocellular carcinoma by integrated bioinformatics analysis
}

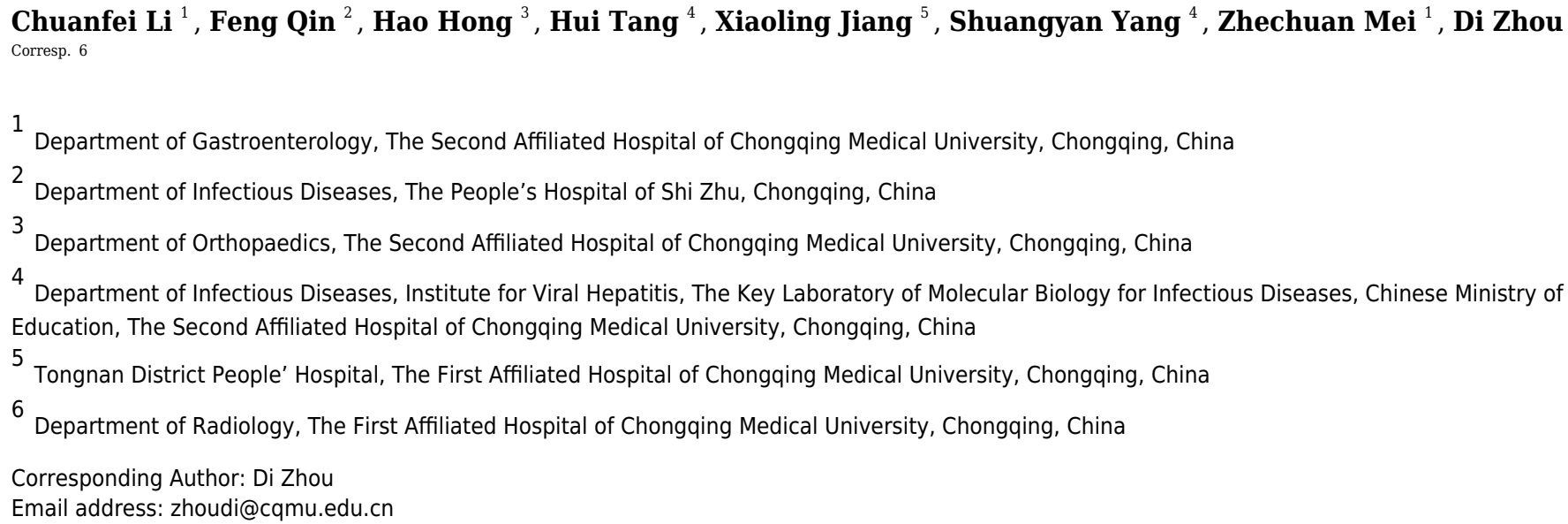

Hepatocellular carcinoma (HCC) is a common yet deadly form of malignant cancer. However, the specific mechanisms involved in HCC diagnosis have not yet fully elucidated. Herein, we screened four publically available Gene Expression Omnibus (GEO) expression profiles ( GSE14520, GSE29721, GSE45267 and GSE60502), and used them to identify 409 differentially expressed genes (DEGs), including 142 and 267 up- and down-regulated genes, respectively. The DAVID database was used to look for functionally enriched pathways among DEGs, and the STRING database and Cytoscape platform were used to generate a protein-protein interaction (PPI) network for these DEGs. The cytoHubba plug-in was utilized to detect 185 hub genes, and three key clustering modules were constructed with the MCODE plug-in. Gene functional enrichment analyses of these three key clustering modules were further performed, and nine core genes including BIRC5, DLGAP5, DTL, FEN1, KIAA0101, KIF4A, MCM2, MKI67, and RFC4, were identified in the most critical cluster. Subsequently, the hierarchical clustering and expression of core genes in TCGA liver cancer tissues were analyzed using the UCSC Cancer Genomics Browser, and whether elevated core gene expression was linked to a poor prognosis in HCC patients was assessed using the GEPIA database. The PPI of the nine core genes revealed an interaction between FEN1, MCM2, RFC4, and BIRC5. Furthermore, the expression of FEN1 was positively correlated with that of three other core genes in TCGA liver cancer tissues. FEN1 expression in HCC and other tumor types was assessed with the FIREBROWSE and ONCOMINE databases, and results were verified in HCC samples and hepatoma cells. FEN1 
levels were also positively correlated with tumor size, distant metastasis and vascular invasion. In conclusion, we identified nine core genes associated with HCC development, offering novel insight into HCC progression. In particular, the aberrantly elevated FEN1 may represent a potential biomarker for HCC diagnosis and treatment. 
1 Identification of Flap endonuclease 1 as a potential

2 core gene in hepatocellular carcinoma by integrated

3 bioinformatics analysis

4

5

6

7

8

9

10

11

Chuanfei Li ${ }^{1}$, Feng Qin², Hao Hong ${ }^{3}$, Hui Tang ${ }^{4}$, Xiaoling Jiang ${ }^{5}$, Shuangyan Yang ${ }^{4}$, Zhechuan $\mathrm{Mei}^{1}$, Di Zhou ${ }^{6}$

${ }^{1}$ Department of Gastroenterology, The Second Affiliated Hospital of Chongqing Medical University, Chongqing, China

${ }^{2}$ Department of Infectious Diseases, The People's Hospital of Shi Zhu, Chongqing, China

${ }^{3}$ Department of Orthopaedics, The Second Affiliated Hospital of Chongqing Medical University, Chongqing, China

${ }^{4}$ Department of Infectious Diseases, Institute for Viral Hepatitis, The Key Laboratory of Molecular Biology for Infectious Diseases, Chinese Ministry of Education, The Second Affiliated Hospital of Chongqing Medical University, Chongqing, China

${ }^{5}$ Tongnan District People' Hospital, The First Affiliated Hospital of Chongqing Medical University, Chongqing, China

${ }^{6}$ Department of Radiology, The First Affiliated Hospital of Chongqing Medical University, Chongqing, China

Corresponding Author:

Di Zhou

No.1 Youyi Road, Yuzhong District, Chongqing, 400016, China

Email address: zhoudi@cqmu.edu.cn

\section{ABSTRACT}

Hepatocellular carcinoma (HCC) is a common yet deadly form of malignant cancer. However, the specific mechanisms involved in HCC diagnosis have not yet fully elucidated. Herein, we screened four publically available Gene Expression Omnibus (GEO) expression profiles (GSE14520, GSE29721, GSE45267 and GSE60502), and used them to identify 409 differentially expressed genes (DEGs), including 142 and 267 up- and down-regulated genes, respectively. The DAVID database was used to look for functionally enriched pathways among DEGs, and the STRING database and Cytoscape platform were used to generate a proteinprotein interaction (PPI) network for these DEGs. The cytoHubba plug-in was utilized to detect 185 hub genes, and three key clustering modules were constructed with the MCODE plug-in. Gene functional enrichment analyses of these three key clustering modules were further performed, and nine core genes including BIRC5, DLGAP5, DTL, FEN1, KIAA0101, KIF4A, MCM2, MKI67, and RFC4, were identified in the most critical cluster. Subsequently, the 
hierarchical clustering and expression of core genes in TCGA liver cancer tissues were analyzed using the UCSC Cancer Genomics Browser, and whether elevated core gene expression was linked to a poor prognosis in HCC patients was assessed using the GEPIA database. The PPI of the nine core genes revealed an interaction between FEN1, MCM2, RFC4, and BIRC5. Furthermore, the expression of FEN1 was positively correlated with that of three other core genes in TCGA liver cancer tissues. FEN1 expression in HCC and other tumor types was assessed with the FIREBROWSE and ONCOMINE databases, and results were verified in HCC samples and hepatoma cells. FEN1 levels were also positively correlated with tumor size, distant metastasis and vascular invasion. In conclusion, we identified nine core genes associated with HCC development, offering novel insight into HCC progression. In particular, the aberrantly elevated FEN1 may represent a potential biomarker for HCC diagnosis and treatment.

Subjects Bioinformatics, Gastroenterology and Hepatology

Keywords Hepatocellular carcinoma, Core genes, Bioinformatics analysis, Flap endonuclease 1

\section{INTRODUCTION}

Hepatocellular carcinoma remains among the most common and deadly forms of cancer globally, posing a significant threat to human life (Forner et al. 2018). HCC and other tumors develop as the result of the long-term accumulation of genetic mutations. Although a large number of biomarkers for the diagnosis of HCC have been identified (C et al. 2018), the specific molecular mechanisms related to the onset, recurrence and treatment of $\mathrm{HCC}$ remain obscure. Therefore, it is essential to identify and exploit novel biomarkers involved in HCC onset and progression to better understand the pathogenesis of HCC.

Between human genome sequencing efforts and the rapid development of gene sequencing technologies, precision medicine has risen to prominence and been widely employed in the field of oncology (IR et al. 2017; $\mathrm{R}$ et al. 2019). Precision medicine relies upon initially exploring potential therapeutic targets via high-throughput sequencing technologies (PN, 2012), as these technologies allow for the large-scale investigation of altered gene expression in the context of disease (Chen et al. 2010). However, sequencing results are often limited and inconsistent owing to the heterogeneity of samples in independent studies, and due to the fact that most studies focus on one cohort. As such, this study sought to analyze genes involved in liver cancer development using a range of available liver cancer-related gene chip datasets, with the goal of identifying potential novel molecular targets for liver cancer treatment and diagnosis.

For the purposes of this study, four HCC related Gene Expression Omnibus (GEO) database datasets were downloaded: GSE14520 (S et al. 2012; S et al. 2010), GSE29721 (B et al. 2011), GSE45267 (CL et al. 2018) and GSE60502 (YH et al. 2014). By analyzing these four datasets, we identified 409 DEGs including 142 up-regulated and 267 down-regulated genes. The DAVID database was then used for a functional enrichment analysis of these DEGs, while the STRING database and Cytoscape were utilized to generate a protein-protein interaction (PPI) network, and three clustering modules were filtered out with the MCODE plug-in, among which clustering 
79

80

81

82

83

84

85

86

87

88

89

90

91

92

93

94

95

96

97

98

99

100

101

102

103

104

105

106

107

108

109

110

111

112

113

114

115

116

117

118

module 1 was most associated with HCC. In addition, nine core genes including BIRC5, DLGAP5, DTL, FEN1, KIAA0101, KIF4A, MCM2, MKI67, and RFC4, were identified within clustering module 1 , and these corresponded to the hub genes in our PPI network. Using the GEPIA database, we performed survival analyses of patients based on expression of these nine core genes, revealing that their overexpression was linked to a poorer prognosis in HCC patients. When we surveyed the literature surrounding these genes, we found that all except for FEN1 had previously been confirmed to play a vital role in HCC. Existing studies have shown that FEN1 is highly expressed in various cancers, such as brain (Nikolova et al. 2009), lung (He et al. 2017; Zhang et al. 2018), breast (Abdel-Fatah et al. 2014), gastric (Wang et al. 2014), prostate (Lam et al. 2006) and pancreatic cancer (Isohookana et al. 2018), but its expression and role in HCC remains unclear. Our previous data indicated that FEN1 expression was elevated in HCC tumors, and this was confirmed upon comparing HCC samples and hepatoma cells to appropriate controls. Together, the results of these analyses suggest that FEN1 may be a core gene orchestrating the progression of HCC.

\section{MATERIALS \& METHODS}

\section{Data collection and DEG validation}

Four liver cancer-related datasets (GSE14520, GSE29721, GSE45267 and GSE60502), were downloaded from GEO (http://www.ncbi.nlm.nih.gov/geo/). These datasets contained a total of 299 tumor samples and 289 non-tumor samples. GSE14520 contained 225 liver cancer samples and 220 adjacent controls; GES29721 contained 10 pairs of liver cancer samples and adjacent control tissue; GSE45267 contained 46 liver cancer samples and 41 adjacent controls; and GSE60502 contained 18 pairs of liver cancer tissues and adjacent controls. The inclusive criteria for datasets were as follows: (1) the samples were from human HCC tissues and paired adjacent or non-tumor tissues; (2) gene expression profiling of mRNA; (3) each dataset included no less than ten paired samples; (4) the datasets were from 2009-2019. The exclusive criteria for datasets were as follows: (1) the HCC samples were induced by specific diseases, including chronic hepatitis $\mathrm{B}$, hepatitis $\mathrm{C}$, hepatitis $\mathrm{D}$, alcoholic liver disease, nonalcoholic fatty liver disease and cholangiocarcinoma; (2) Details of the samples were not available; (3) the datasets could not be analyzed by GEO2R; (4) No gene symbol. The specific platform information for these four datasets is compiled in Table 1. GEO2R was utilized to identify DEGs in these studies, using the screening criteria: $\mid \log \mathrm{FC}$ (fold change) $\mid \geqslant 1, \mathrm{P}<0.05$, and adjusted $\mathrm{P}<0.05$. In addition, genes with multiple probe set or probe sets lacking matched gene symbols were removed or averaged, respectively. Then, the overall DEGs, as well as those that were up- and downregulated in the four datasets were intersected and visualized using Funrich (v 3.0, http://funrich.org/index.html).

\section{Functional enrichment analyses}

Gene ontology analyses focuses on three domains: biological processes (BP), cellular components (CC), and molecular functions (MF), and such analyses are commonly used to understand the biological functions, pathways, or localization of DEGs. The Kyoto Encyclopedia 
119

120

121

122

123

124

125

126

127

128

129

130

131

132

133

134

135

136

137

138

139

140

141

142

143

144

145

146

147

148

149

150

151

152

153

154

155

156

157

158

of Genes and Genomes (KEGG) pathway analysis database surveys as a valuable resource for assessing how particular DEGs may be involved in or influenced by specific signaling pathways and disease states. The DAVID (https://david.ncifcrf.gov/) was utilized for functional enrichment analyses, with a $\mathrm{P}<0.05$ cutoff for significance.

\section{PPI network analysis}

The STRING database (v10.5; https://stringdb.org/) was used to generate a DEG PPI, with a minimum interaction score cutoff of 0.4. Cytoscape (v 3.4.0, https://cytoscape.org/) was used for network visualization, and the CytoHubba plug-in was used to identify hub genes with the criteria of filtering degree $\geqslant 10$. The MCODE plug-in was used to construct key clustering modules $($ MCODE score $>10$, degree cut-off $=2$, node score cut-off $=0.2$, Max depth $=100$ and $\mathrm{k}$-score $=2$ ).

\section{Validation of core genes}

Hub genes among overall DEGs and the most critical clustering module were identified through the CytoHubba plug-in, and intersecting core genes were identified. The UCSC Cancer Genomics Browser (https://genome-cancer.ucsc.edu/) was then used for hierarchical clustering of these core genes. In addition, the expression profiles of these core genes in 421 TCGA liver cancer tissues, including 50 solid normal tissues and 371 primary tumors, were determined by analyzing available datasets. A core gene PPI network was constructed with the cBioportal online database (http://www.cbioportal.org/). Furthermore, correlations between the expression of FEN1 and MCM2, RFC4, or BIRC5 in TCGA liver cancer tissues were investigated. Finally, we used the ONCOMINE (https://www.oncomine.org/resource/login.html) and FIREBROWSE online database (http://firebrowse.org/) to investigate the FEN1 expression in various cancers including $\mathrm{HCC}$.

\section{Survival analysis}

The GEPIA database (http://gepia.cancer-pku.cn/) was used to conduct survival analyses based on core gene expression, with hazard ratios (HRs) and 95\% confidence intervals being calculated, and logrank $\mathrm{P}$ value $<0.05$ being the threshold of statistical significance.

\section{Clinical samples}

A total of 34 paired HCC tumor tissues as well as corresponding adjacent non-cancerous tissues were obtained from our Hospital's Department of Hepatobiliary Surgery, with all patients providing informed consent. The content of the informed consent includes research purposes, risks and discomfort, benefits, and privacy issues. The form of the informed consent is in written. The study was examined and approved by the Second affiliated Hospital of Chongqing Medical University Ethics Committee (approval number: 2018-60).

\section{Cell culture}


159 Seven human liver cancer cell lines (SMMC-7721, BEL-7404, HCCLM3, HepG2, MHCC97-H, 160 SK-HEP-1, and Huh-7) as well as normal human liver HL7702 cells were donated by the 161 Institute for Viral Hepatitis, Chongqing Medical University. All cells were grown in high 162 glucose DMEM (Gibco, USA) containing $10 \% \mathrm{FBS}$ (Corning, USA) at $37^{\circ} \mathrm{C}$ in a $5 \% \mathrm{CO}_{2}$ 163 incubator.

164

\section{Hematoxylin and eosin $(\mathrm{H} \& \mathrm{E})$ staining}

166 Paraffin-embedded tissues were dewaxed in xylene I, II, and III for 20 minutes each, and then

167

168

169

170

171

172

173

174

175

176

177

178

179

180

181

182

183

184

185

186

187

188

189

190

191

192

193

194

195

196

197

198 dehydrated in an ethanol gradient $(100 \%, 95 \%, 90 \%, 80 \%$, and $70 \%), 3$ minutes per step.

Sections were then rinsed with distilled water for 5 minutes, and the nucleus was counterstained with hematoxylin for 3 minutes. Sections were washed again in water, followed by differentiation for 30 seconds in a $75 \%$ hydrochloric acid alcohol solution, and blue color was returned by washing with distilled water for 5 minutes. A red dye was then used for counterstaining for 5 minutes, after which samples were dehydrated in $70 \%, 80 \%, 95 \%$, and $100 \%$ ethanol, 1 minute per concentration. Sections were then cleared with xylene and sealed using neutral gum.

\section{IHC analysis}

IHC staining was conducted as previously described (WG et al. 2016). Briefly, paraffinembedded sections were incubated at $56^{\circ} \mathrm{C}$ for $2 \mathrm{~h}$, and then $3 \%$ hydrogen peroxide was used for antigen retrieval. Afterwards, the sections were incubated with rabbit anti-human FEN1 (1:100, A1175, ABclonal, China) at $4^{\circ} \mathrm{C}$ overnight. Then, sections were probed for $1 \mathrm{~h}$ using HRPconjugated secondary antibodies at $37^{\circ} \mathrm{C}$, after which a DAB substrate kit was utilized, and hematoxylin was used for nuclear staining. The Image-Pro Plus (IPP) software (Media Cybernetics, Rockville, MD, USA) was used to quantify staining intensity. The frequency of positively-stained cells was determined on a $0-100$ scale, while staining intensity was scored as follows: $0=$ negative; $1=$ weak; $2=$ moderate; $3=$ strong. These two scores were then multiplied together to yield an IHC score between 0 and 300 . The final scores were assigned by two independent pathologists. The mean IHC score was used as a cutoff value to separate patients into low- and high-expression groups.

\section{RT-qPCR analysis}

TRIzol (ThermoFisher Scientific, USA) was used for total RNA extraction, and RNA was then reverse transcribed with the PrimeScript RT-PCR kit (Takara Bio, Dalian, China) based on provided protocols. The SYBR Premix Ex Taq II (Takara, Japan) kit was used to conduct RTqPCR analysis on a Bio-Rad CFX96 Real-Time System (Bio-Rad, Hercules, CA). The reaction conditions were as follows: pre-denaturation at $95^{\circ} \mathrm{C}$ for $30 \mathrm{~s}$, followed by 40 cycles of amplification at $95^{\circ} \mathrm{C}$ for $5 \mathrm{~s}$ and $60^{\circ} \mathrm{C}$ for $30 \mathrm{~s}$, and a final cycle along the melting curve from $65^{\circ} \mathrm{C}$ to $95^{\circ} \mathrm{C}$ in increments of $0.5^{\circ} \mathrm{C}$ for $5 \mathrm{~s}$. mRNA levels were determined via the $2^{-\Delta \Delta \mathrm{Ct}}$ method, with GAPDH used for normalization. Primers used were: GAPDH F: 5'- 
199

200

201

202

203

204

205

206

207

208

209

210

211

212

213

214

215

216

217

218

219

220

221

222

223

224

225

226

227

228

229

230

231

232

233

234

235

236

237

GGTGGTCTCCTCTGACTTCAACA -3' and R: 5'- GTTGCTGTAGCCAAATTCGTTGT-3', FEN1 F: 5'- CTGTGGACCTCATCCAGAAGCA -3' and R: 5'CCAGCACCTCAGGTTCCAAGA -3'.

\section{Statistical analysis}

Statistical analyses and graphing were performed with SPSS v19.0 (SPSS Inc., USA) and Graph Pad Prism v8.0 (Graph Pad Software, USA), respectively. Data are means \pm standard deviation (SD). Student's t-tests were used to compare groups. Fisher's exact test was used to assess correlations between the expression of FEN1 and HCC patient clinicopathological features. Spearman's correlation analyses were used to compare the expression of pairs of genes in TCGA liver cancer tissues. $\mathrm{P}<0.05$ was the significance threshold $\left(* \mathrm{P}<0.05,{ }^{*} \mathrm{P}<0.01\right)$.

\section{RESULTS}

\section{HCC-associated DEG identification}

In this study, 1088 total DEGs (505 and 583 up- and down-regulated, respectively) in GSE14520, 1449 total DEGs (837 and 612 up- and down-regulated, respectively) in GSE29721, 1604 total DEGs (713 and 891 up- and down-regulated, respectively) in GSE45267, and 1533 total DEGs (792 and 741 up- and down-regulated, respectively) in GSE60502 were screened. Based on these datasets, a total of 409 overlapping DEGs were identified among these four datasets (142 and 267 up- and down-regulated, respectively), as visualized with the Funrich software (Figure 1). DEGs are listed in Table S1.

\section{DEG functional enrichment analyses}

To explore the biological activities of these DEGs, the DAVID database was used to conduct GO and KEGG enrichment analysis. With respect to BPs, up-regulated DEGs were primarily enriched in processes such as mitotic nuclear division, cell division, cell cycle, DNA replication, and mitotic sister chromatid segregation (Figure S1A), while down-regulated DEGs were primarily enriched in processes such as redox process, the cytochrome 450 pathway, drug metabolism, and negative regulation of growth (Figure S2A). With respect to CCs, up-regulated DEGs were primarily enriched in the nucleoplasm, nucleus, cytoplasm, spindle, and cellular intermediates (Figure S1B), while down-regulated DEGs were mostly enriched in extracellular exosomes, organelle membranes, blood microparticles, extracellular regions, and the mitochondrial matrix (Figure S2B). With respect to MFs, up-regulated DEGs were primarily enriched in functions such as protein binding, ATP binding, DNA helicase activity, protein kinase binding, single-stranded DNA binding, and chromatin binding (Figure S1C), whereas down-regulated DEGs were primarily associated with iron ion binding, oxidoreductase activity, heme binding, monooxygenase activity, and oxygen binding (Figure S2C).

A KEGG analysis revealed that up-regulated DEGs were particularly enriched in pathways such as the cell cycle, DNA replication, P53 signaling, and tumor pathways (Figure S1D). 
238 However, down-regulated DEGs were mostly associated with metabolic pathways, fatty acid

239

240

241

242

243

244

245

246

247

248

249

250

251

252

253

254

255

256

257

258

259

260

261

262

263

264

265

266

267

268

269

270

271

272

273

274

275

276

277

degradation, chemical carcinogenesis, and PPAR signaling (Figure S2D).

\section{PPI network and module analyses}

To better understand interactions among DEGs, the STRING online database was used to generate a PPI network consisting of 403 nodes and 3502 edges, which was visualized using Cytoscape. Six of the 409 DEGs were not included in this network (Figure 2A). This network was then analyzed using the MCODE plug-in, and three clustering modules were filtered out according to the chosen screening conditions. Clustering module 1 scored 58.492 with 62 nodes and 1784 edges (Figure 2B), clustering module 2 scored 11.529 with 18 nodes and 98 edges (Figure 2C), and clustering module 3 scored 10.917 with 25 nodes and 131 edges (Figure 2D). The genes in clustering module 1 were up-regulated DEGs, whereas those in the other two modules were primarily down-regulated DEGs.

\section{Functional enrichment analysis of key clustering modules}

The DAVID database was next used to explore the biological functions of genes in these key clustering modules (Tables S2-4). With respect to BPs, clustering module 1 was primarily enriched in cell differentiation, mitotic nuclear division, DNA replication, and DNA helicase activity, while clustering module 2 was primarily enriched in plasminogen activation, coagulation, cytolysis, and complement activation regulation, and clustering module 3 was primarily enriched in steroid metabolism, heterogeneous biomass metabolism, and exogenous drug catabolism. With respect to CCs, clustering module 1 was primarily enriched for the nucleoplasm, nucleus, intermediate, spindle, cytoplasm, nuclear chromosome, while clustering module 2 was primarily enriched for exosomes, extracellular regions, membrane attack complexes, and extracellular vesicles, and clustering module 3 was primarily enriched for organelle membranes, the endoplasmic reticulum membrane, and high-density lipoproteins. With respect to MFs, clustering module 1 was primarily enriched for protein binding, ATP binding, DNA helicase activity, protein kinase binding, and DNA binding, while clustering module 2 was primarily enriched for endopeptidase activity, transcription factor binding, steroid binding, RNA polymerase II transcription factor activity, and enzyme binding activity, and clustering module 3 was primarily enriched for aerobic binding, iron ion binding and heme binding.

A KEGG analysis revealed that clustering module 1 was primarily enriched in the cell cycle, oocyte meiosis, DNA replication, and p53 signaling, while clustering module 2 was primarily enriched in the complement system, prion disease, and systemic lupus erythematosus, and clustering module 3 was primarily enriched in chemical carcinogenesis, retinol metabolism, P450 drug metabolism, and metabolic pathways.

\section{Identification of core genes and analysis of their clinical significance}

Next, core genes involved in HCC were identified based on their levels of interaction via analyzing our PPI network using the Cytoscape program. Based on our clustering module 
278 analysis, clustering module 1 included 62 genes was found closely related to the progression of 279 HCC. Then, nine total genes (BIRC5, DLGAP5, DTL, FEN1, KIAA0101, KIF4A, MCM2, 280 MKI67, and RFC4) were identified based on the intersecting genes among the top 40 genes 281 derived from 12 different algorithms by the cytoHubba plug-in (Table S5). In addition, 185 hub

282

283

284

285

286

287

288

289

290

291

292

293

294

295

296

297

298

299

300

301

302

303

304

305

306

307

308

309

310

311

312

313

314

315

316

317

genes were identified across 403 nodes in our PPI network based on the filtering degree $\geqslant 10$ criteria (Table S6). The nine identified core genes were found belonged to this larger subset of hub genes (Figure 3A).

To investigate core gene expression in $\mathrm{HCC}$, a hierarchical clustering analysis was performed using the UCSC Cancer Genomics Browser, revealing that these nine core genes were highly expressed in most liver cancer samples (Figure 3C). Next, the expression profiles of these nine core genes in 421 TCGA liver cancer tissues, including 50 solid normal tissues and 371 primary tumors, were downloaded and analyzed, revealing that the expression of these core genes was significantly elevated in HCC (Figure 3B). The correlations between core gene expression levels and patient prognosis in 182 total $\mathrm{HCC}$ samples were further assessed, revealing that $\mathrm{BIRC} 5$ expression $(\mathrm{HR}=2$, logrank $\mathrm{P}=6.7 \mathrm{e}-05)$ was correlated with worse overall survival (OS) for HCC patients, as was that of DLGAP5 ( $\mathrm{HR}=1.9$, logrank $\mathrm{P}=0.00039)$, DTL $(\mathrm{HR}=1.7, \log \operatorname{rank} \mathrm{P}=0.0049), \mathrm{FEN} 1(\mathrm{HR}=1.5$, logrank $\mathrm{P}=0.022), \mathrm{KIAA} 0101(\mathrm{HR}=1.7$, $\operatorname{logrank} \mathrm{P}=0.002), \mathrm{KIF} 4 \mathrm{~A}(\mathrm{HR}=1.8, \operatorname{logrank} \mathrm{P}=0.001), \mathrm{MCM} 2(\mathrm{HR}=1.7$, logrank $\mathrm{P}=0.0022)$, MKI67 ( $\mathrm{HR}=1.9$, logrank $\mathrm{P}=0.00045)$, and $\mathrm{RFC4}(\mathrm{HR}=1.7$, logrank $\mathrm{P}=0.004)$ (Figure 4).

Furthermore, the expression of BIRC5 $(\mathrm{HR}=1.6$, logrank $\mathrm{P}=0.002)$ was correlated with decreased disease-free survival (DFS) for HCC patients, as was that of DLGAP5 (HR=1.6, $\operatorname{logrank} \mathrm{P}=0.0033)$, DTL $(\mathrm{HR}=1.6$, logrank $\mathrm{P}=0.0016)$, FEN1 (HR=1.5, logrank $\mathrm{P}=0.0075)$, KIAA0101 $(\mathrm{HR}=1.6$, logrank $\mathrm{P}=0.0022), \mathrm{KIF} 4 \mathrm{~A}(\mathrm{HR}=1.6$, logrank $\mathrm{P}=0.0011), \mathrm{MCM} 2(\mathrm{HR}=$ 1.6, logrank $\mathrm{P}=0.0034)$, MKI67 $(\mathrm{HR}=1.9$, logrank $\mathrm{P}=4.2 \mathrm{e}-05)$, and $\mathrm{RFC} 4(\mathrm{HR}=1.5$, logrank $\mathrm{P}=0.011$ ) (Figure 5). High expression of these nine core genes was associated with significantly reduced survival among HCC patients.

\section{FEN1 may be a key candidate gene in HCC}

To clarify the PPI network for these nine core genes, the cBioportal online database were explored and identified an interaction between FEN1, MCM2, BIRC5 and RFC4 (Figure 6A). Previous studies have confirmed that MCM2, BIRC5, and RFC4 are abnormally highly expressed in HCC, and that they participate in the regulation of HCC tumor biology. However, the expression of FEN1 and its clinical significance in HCC is unclear. Therefore, the correlations between the expression of FEN1 and these three other genes were further analyzed. The expression of FEN1 in TCGA liver cancer tissues was positively correlated with that of MCM2 ( $\mathrm{r}=0.853, \mathrm{P}=0.000)$, BIRC5 ( $\mathrm{r}=0.809, \mathrm{P}=0.000)$, and RFC4 $(\mathrm{r}=0.852, \mathrm{P}=0.000)$ (Figure 6B-D), suggesting that FEN1 may play as important a role in the progression of liver cancer as do MCM2, BIRC5, and RFC4. The ONCOMINE and FIREBROWSE databases were further utilized to investigate the expression of FEN1 in various cancers, including HCC. The results revealed that FEN1 expression was clearly elevated in most cancers, including bladder,

Peer) reviewing PDF | (2019:06:38406:1:1:NEW 1 Aug 2019) 
318 breast, colorectal, esophageal, lung, and liver cancer (Figure 7A, B). In addition, FEN1 was

319 found overexpressed in three HCC-related datasets (Figure 7C).

320

321

322

323

324

325

326

327

328

329

330

331

332

333

334

335

336

337

338

339

340

341

342

343

344

345

346

347

348

349

350

351

352

353

354

355

356

357

\section{Experimental validation}

In this study, 34 paired HCC and adjacent control tissues were used to verify the expression of FEN1 in HCC. The pathologic diagnosis of HCC and matched adjacent samples were confirmed by $\mathrm{H} \& \mathrm{E}$ staining (Figure 8A, C). FEN1 staining was localized to the nucleus and cytoplasm, with stronger expression in the HCC samples relative to the adjacent controls (Figure 8B, D). Furthermore, IHC analysis indicated that FEN1 levels were significantly higher in HCC relative to adjacent tissues (Figure 8E), with IHC scores being significantly higher in $\mathrm{HCC}$ samples (Figure 8F).

The correlation between FEN1 expression and HCC patient clinicopathological features was investigated via Fisher's exact test. As shown in Table 2, there were significant correlations between FEN1 expression and tumor size $(\mathrm{P}=0.047<0.05)$, metastasis $(\mathrm{P}=0.013<0.05)$ and vascular invasion $(\mathrm{P}=0.024<0.05)$. $\mathrm{FEN} 1$ expression did not significantly correlated with gender, age, tumor multiplicity, TNM stage, pathological grade, HBsAg, liver cirrhosis or serum alpha-fetoprotein (AFP) $(\mathrm{P}>0.05)$. Furthermore, FEN1 mRNA levels were found significantly elevated in six human hepatoma cell lines relative to that in the normal human liver cell line HL7702 (Figure 8G).

\section{DISCUSSION}

The occurrence and progression of HCC are complex, with multiple cumulative genetic changes ultimately culminating in progressive disease. High-throughput technologies such as gene chips have been widely employed to elucidate the underlying mechanisms, providing an innovative and effective approach to the diagnosis, prevention, and treatment of HCC. Numerous studies have been conducted to clarify genetic changes underlying HCC development, but results to date remain inconclusive of incomplete. As such, there is further need to investigate the molecular mechanisms governing HCC.

Functional analysis of DEGs and screening of core genes: In this study, we identified 409 total DEGs (142 and 267 up- and down-regulated, respectively) shared among four HCC datasets, and these genes were used for functional enrichment analyses. A GO analysis revealed the up-regulated DEGs to be primarily linked with cell division, the cell cycle, and DNA replication, whereas down-regulated DEGs were mostly associated with redox reactions, cytochrome 450 functionality, and negative growth regulation. A KEGG pathway analysis revealed up-regulated DEGs to mostly be associated with signaling relating to the cell cycle, DNA replication, p53 signaling, and tumor pathways, whereas down-regulated DEGs were mostly linked to metabolic pathways such as fatty acid degradation. These results suggested that the up-regulated DEGs may affect HCC progression via regulating DNA replication and the cell cycle, whereas down-regulated DEGs may be linked to HCC progression through metabolic pathways. Previous studies have indicated that the dysregulation of the cell cycle is a key

Peer) reviewing PDF | (2019:06:38406:1:1:NEW 1 Aug 2019) 
358

359

360

361

362

363

364

365

366

367

368

369

370

371

372

373

374

375

376

377

378

379

380

381

382

383

384

385

386

387

388

389

390

391

392

393

394

395

396

397

hallmark of many cancer types (P et al., 2017). As one of the most important tumor suppressor genes, p53 is closely related to tumorigenesis, with at least $50 \%$ of cancer patients exhibiting p53 mutations or loss of function (C et al. 2013; G \& M 2019; MP et al. 2015). P53 signaling dysregulation has repeatedly been confirmed to be linked with cancer development (M \& J 2016). There is also increasing evidence that metabolism regulates cancer growth and proliferation (J \& CB 2019; T et al. 2016). We further used our DEGs to generate a PPI, which consisted of three key clustering modules screened using the MCODE plug-in. Functional enrichment analyses revealed that clustering module 1 was closely related to gene mutations in the progression of HCC, and was enriched in genes linked to the cell cycle, DNA replication, and the p53 signaling pathway. Then nine core genes (BIRC5, DLGAP5, DTL, FEN1, KIAA0101, KIF4A, MCM2, MKI67 and RFC4) in clustering module 1 were screened, as these genes were also hub genes in the overall PPI network. There are numerous studies related to HCC chips that have sought to identify the molecules involved in the onset and progression of HCC. However, due to the different data sets and search strategies included in each study, the key molecules identified are different. Nevertheless, the core genes we identified were similar to those found by other researchers. Cai C (Cai, Wang, \& Tu, 2019) identified twelve hub gene including BIRC5 and MCM2 in HCC. DTL and MCM2 were also identified as hub genes for HCC in another study (Sang, Wang, Xu, \& Zhao, 2018). Shen S (Shen et al., 2019) identified FEN1 and RFC4 as core genes by analyzing four HCC datasets. The above studies indicated the core genes screened and identified in our study were of value. Afterwards, the hierarchical clustering and expression profiles of core genes in TCGA liver cancer tissues were analyzed using the UCSC Cancer Genomics Browser, and survival analyses suggested that aberrantly high expression of these core genes was predictive of a poor HCC patient prognosis, suggesting these core genes may be key molecular biomarkers for HCC diagnosis and treatment.

Research progress of core genes in HCC: BIRC5, also known as survivin, is an antiapoptotic protein reported to function as a potential oncogene in the context of many cancers (MJ et al. 2007). Studies have shown that BIRC5 is highly expressed in the vast majority of tumors, including HCC (C 2016). In addition, elevated BIRC5 levels have been found to be associated with histological grade, tumor size, and TNM stage in HCC patients (W et al. 2019). DLGAP5, also known as HURP, is a cell cycle regulatory gene that has been found to be highly expressed in liver cancer (Chang et al. 2011; Tsou et al. 2003). Weijia Liao (Liao et al. 2013) reported the abnormally high expression of DLGAP5 in HCC, and found that this was related to promoter methylation level, with the silencing of DLGAP5 significantly inhibiting cell proliferation, migration, and colony formation in vitro. DLGAP5 knockdown inhibited the proliferation of hepatoma cells by reducing P53 accumulation (Kuo et al. 2012). DTL is a substrate receptor for the CRL4 ubiquitin ligase, serving as a key regulator of the cell cycle and genomic stability. DTL upregulation in invasive HCC has been found to be positively correlated with tumor grade and patient survival (Chen et al. 2018b; Pan et al. 2006). FEN1, a structurally specific metal nuclease, plays a vital role in DNA damage repair (Lieber 1997) and maintenance of genomic stability (Becker et al. 2018). FEN1 has been found to be expressed at high levels in several 
398

399

400

401

402

403

404

405

406

407

408

409

410

411

412

413

414

415

416

417

418

419

420

421

422

423

424

425

426

427

428

429

430

431

432

433

434

435

436

437

cancer types, including those of the lung (He et al. 2017), breast (Abdel-Fatah et al. 2014), gastric (Wang et al. 2014), prostate (Lam et al. 2006) and pancreatic (Isohookana et al. 2018). FEN1 mutations have been associated with the occurrence of gastrointestinal tumors, including HCC (Liu et al. 2012b), suggesting that FEN1 may be important in the development of gastrointestinal tumors. Nevertheless, the expression and biological function of FEN1 in HCC remains unclear. KIAA0101 is a nuclear antigen-associated factor that is present in proliferating cells and which regulates proliferative processes. KIAA0101 has been found to be highly expressed in $\mathrm{HCC}$, representing a potential biomarker for this tumor type that is relevant to disease treatment and patient prognosis (Yuan et al. 2007). KIAA0101 variant 1 can promote the survival of liver cancer cells by regulating p53 functionality, indicating that inhibition of KIAA0101 variant 1 may be a promising therapeutic strategy (Liu et al. 2012a). Numerous studies have revealed that KIF4A is an oncogene in the context of lung, oral, and breast cancer. High KIF4A expression was significantly correlated with tumor stage, tumor differentiation, and metastasis, and may be a biomarker for poor HCC prognosis (Hou et al. 2017). In addition, KIF4A promoted the proliferation and invasion of hepatoma cells via p53 and Akt signaling (Hou et al. 2017; Huang et al. 2018). MCM2 has been shown to be critical for chromatin composition and for the focal formation of p53 binding protein 1 in HepG2 cells (Chen et al. 2018a). High MCM2 expression has been associated with poor prognosis in HCC patients (Liu et al. 2018). MKI67 encodes a nuclear antigen expressed during the G1, S and G2-M phases in proliferating cells, and its expression levels are closely related to tumor growth rate ( $\mathrm{T}$ et al. 1995), histological stage (IO et al. 1995), and tumor recurrence for HCC (K et al. 1995). Higher MKI67 expression was associated with both faster HCC progression and a poorer patient prognosis ( $Y$ et al. 2015). Consistent with our results, RFC4 has been found to be overexpressed in HCC (M et al. 2009). The silencing of RFC4 expression was able to reduce HepG2 cell proliferation and promote apoptosis, and was also associated with the increased sensitivity of cells to doxorubicin and camptothecin, suggesting RFC4 may be a novel target for liver cancer treatment.

The identification of FEN1 and its potential mechanisms in HCC: As these past results show, all of these core genes except for FEN1 have been reported to play a role in HCC progression. To further explore interactions among these nine core genes, a PPI network was generated revealing interactions between FEN1, MCM2, RFC4, and BIRC5. As expected, FEN1 expression was significantly positively correlated with that of MCM2, RFC4, and BIRC5 in TCGA liver cancer tissues, suggesting that FEN1 may be as involved in the development of HCC as are MCM2, RFC4, and BIRC5. Previous studies had confirmed that FEN1 was aberrantly up-regulated in various cancers. However, the expression of FEN1 in HCC remain obscure. Therefore, we first utilized the ONCOMINE and FIREBROWSE online databases to investigate the expression of FEN1 in HCC, and found that FEN1 was highly expressed in multiple tumor tissues including those of HCC patients, consistent with previous reports. We then verified this in HCC tissues from 34 patients via IHC staining. Interestingly, a correlation analysis revealed that high expression of FEN1 was significantly correlated with tumor size, 
438 metastasis and vascular invasion, suggesting that FEN1 may be involved in the regulation of 439 liver cancer proliferation and metastasis. Similarly, a study had confirmed that the expression of 440 FEN1 in gastric cancer was positively associated with the degree of differentiation, lymphatic 441 metastasis, tumor size and TNM stage ( $\mathrm{K}$ et al. 2014). In addition, the FEN1 was up-regulated in 442 lung cancer cells and associated with the clinical lung cancer stage (L et al. 2017). Another study 443 also suggested that FEN1 might contribute to nodal metastasis in lung adenocarcinoma and result 444 in a poor prognosis (JC et al. 2015). These findings suggested that FEN1 may be involved in the regulation of multiple tumor biological processes. Mechanistically, FEN1, a structure-specific metallonuclease, is involved in DNA replication, DNA synthesis, DNA damage repair, nonhomologous end joining and homologous recombination (Lieber 1997; Wu et al. 1999). Importantly, FEN1 is essential for maintaining genome stability (Becker et al. 2018). The upstream and downstream molecules of FEN1 may be involved in the regulation of various tumors. A study has previously reviewed three underlying mechanisms by which FEN1 may be involved in tumorigenesis ( $\mathrm{L}$ et al. 2011). First, the interactions between FEN1 and other DNA metabolic proteins are formed. Second, the super-accumulated FEN1 in nucleolus and mitochondrion is thought to play a vital role in maintaining the stability of tandem ribosomal DNA repeats and in mitochondrial DNA replication. Third, FEN1 could be post-translationally modified through acetylation, methylation, or phosphorylation, and this was important for its ability to regulate nuclease activities and protein partner selection. One study reported that FEN1 knockdown was able to induce DNA damage and enhance the activation of p53 in lung cancer cells (L et al. 2017). Furthermore, FEN1 was found be regulated by the transcriptional repressor Ying Yang 1 (YY1) in response to DNA damaging agents in the context of breast cancer. Another study demonstrated that FEN1 promoted breast cancer cell proliferation via an epigenetic mechanism whereby FEN1-mediated up-regulation of DNMT1 and DNMT3a (X et al. 2019). At present, whether FEN1 participates in HCC progression and what the underlying mechanisms of such participation may be remains unclear. We speculate that it may be related to the above mechanisms. In addition, our results showed that FEN1 was elevated in six human hepatoma cell lines relative to a control cell line via RT-qPCR analysis, which provided some basis for further study of the function of FEN1 in liver cancer. However, the specific biological function of FEN1 in HCC remains to be further investigated.

\section{CONCLUSIONS}

In summary, we screened 409 HCC-associated DEGs, of which 142 and 267 were up- and downregulated, respectively. We identified nine core genes, including BIRC5, DLGAP5, DTL, FEN1, KIAA0101, KIF4A, MCM2, MKI67, and RFC4, that were up-regulated in HCC and that may play important roles in the development or progression of this cancer. We further confirmed the expression of FEN1 in HCC, suggesting that FEN1 may have potential as a new biomarker for HCC diagnosis, treatment, or prognosis determination. Our results offer significant improvements to current understanding of HCC pathogenesis. Additional studies will be needed to validate our findings, and to confirm whether and how FEN1 regulates HCC. 
478

479

480

481

482

483

484

485

486

487

488

489

490

491

492

493

494

495

496

497

498

499

500

501

502

503

504

505

506

507

508

509

510

511

512

513

514

515

516

517

518

519

520

521

522

\section{ACKNOWLEDGEMENTS}

None.

\section{REFERENCES}

Abdel-Fatah TM, Russell R, Albarakati N, Maloney DJ, Dorjsuren D, Rueda OM, Moseley P, Mohan V, Sun H, Abbotts R, Mukherjee A, Agarwal D, Illuzzi JL, Jadhav A, Simeonov A, Ball G, Chan S, Caldas C, Ellis IO, Wilson DM, 3rd, and Madhusudan S. 2014. Genomic and protein expression analysis reveals flap endonuclease 1 (FEN1) as a key biomarker in breast and ovarian cancer. Mol Oncol 8:1326-1338. DOI 10.1016/j.molonc.2014.04.009.

B S, J H, B B, M S, M H, ZG H, and M S. 2011. Definition of the landscape of promoter DNA hypomethylation in liver cancer. Cancer Res 71:5891-5903. DOI 10.1158/00085472.CAN-10-3823.

Becker JR, Gallo D, Leung W, Croissant T, Thu YM, Nguyen HD, Starr TK, Brown GW, and Bielinsky AK. 2018. Flap endonuclease overexpression drives genome instability and DNA damage hypersensitivity in a PCNA-dependent manner. Nucleic Acids Res 46:5634-5650. DOI 10.1093/nar/gky313.

C K, MD M, F V, K Y, B N, C L, M X, Q Z, JF M, MA W, MDM L, CA M, JS W, MJ W, MC W, TJ L, RK W, BJ R, and L D. 2013. Mutational landscape and significance across 12 major cancer types. Nature 502:333-339. DOI 10.1038/nature12634.

C S. 2016. Survivin in survival of hepatocellular carcinoma. Cancer Lett 379:184-190. DOI 10.1016/j.canlet.2015.06.016.

C T, A S, and RS H. 2018. Cancer genetics, precision prevention and a call to action. Nat Genet 50:1212-1218. DOI 10.1038/s41588-018-0202-0.

Cai C, Wang W, and Tu Z. 2019. Aberrantly DNA Methylated-Differentially Expressed Genes and Pathways in Hepatocellular Carcinoma. J Cancer 10:355-366. DOI $10.7150 /$ jca. 27832 .

Chang ML, Lin SM, and Yeh CT. 2011. HURP expression-assisted risk scores identify prognosis distinguishable subgroups in early stage liver cancer. PLoS One 6:e26323. DOI 10.1371/journal.pone.0026323.

Chen CF, Hsu EC, Lin KT, Tu PH, Chang HW, Lin CH, Chen YJ, Gu DL, Lin CH, Wu JY, Chen YT, Hsu MT, and Jou YS. 2010. Overlapping high-resolution copy number alterations in cancer genomes identified putative cancer genes in hepatocellular carcinoma. Hepatology 52:1690-1701. DOI 10.1002/hep.23847.

Chen Y, Weng C, Zhang H, Sun J, and Yuan Y. 2018a. A Direct Interaction Between P53Binding Protein 1 and Minichromosome Maintenance Complex in Hepg2 Cells. Cell Physiol Biochem 47:2350-2359. DOI 10.1159/000491607.

Chen YC, Chen IS, Huang GJ, Kang CH, Wang KC, Tsao MJ, and Pan HW. 2018b. Targeting DTL induces cell cycle arrest and senescence and suppresses cell growth and colony formation through TPX2 inhibition in human hepatocellular carcinoma cells. Onco Targets Ther 11:1601-1616. DOI 10.2147/ott.s147453.

CL C, YS T, YH H, YJ L, YY S, CW S, GY C, YC Y, YS C, JT H, and JC W. 2018. Lymphoid Enhancer Factor 1 Contributes to Hepatocellular Carcinoma Progression Through Transcriptional Regulation of Epithelial-Mesenchymal Transition Regulators and 
523

524

525

526

527

528

529

530

531

532

533

534

535

536

537

538

539

540

541

542

543

544

545

546

547

548

549

550

551

552

553

554

555

556

557

558

559

560

561

562

563

564

565

566

567

568

Stemness Genes. Hepatol Commun 2:1392-1407. DOI 10.1002/hep4.1229.

Forner A, Reig M, and Bruix J. 2018. Hepatocellular carcinoma. Lancet 391:1301-1314. DOI 10.1016/s0140-6736(18)30010-2.

G DO, and M C. 2019. Mutant p53 and Cellular Stress Pathways: A Criminal Alliance That

Promotes Cancer Progression. Cancers (Basel) 11: E614. DOI 10.3390/cancers1 1050614.

He L, Luo L, Zhu H, Yang H, Zhang Y, Wu H, Sun H, Jiang F, Kathera CS, Liu L, Zhuang Z, Chen H, Pan F, Hu Z, Zhang J, and Guo Z. 2017. FEN1 promotes tumor progression and confers cisplatin resistance in non-small-cell lung cancer. Mol Oncol 11:640-654. DOI 10.1002/1878-0261.12058.

Hou G, Dong C, Dong Z, Liu G, Xu H, Chen L, Liu L, Wang H, and Zhou W. 2017. Upregulate KIF4A Enhances Proliferation, Invasion of Hepatocellular Carcinoma and Indicates poor prognosis Across Human Cancer Types. Sci Rep 7:4148. DOI 10.1038/s41598-01704176-9.

Huang Y, Wang H, Lian Y, Wu X, Zhou L, Wang J, Deng M, and Huang Y. 2018. Upregulation of kinesin family member 4A enhanced cell proliferation via activation of Akt signaling and predicted a poor prognosis in hepatocellular carcinoma. Cell Death Dis 9:141. DOI 10.1038/s41419-017-0114-4.

IO N, J N, EC L, ST F, and M N. 1995. Ki-67 antigen expression in hepatocellular carcinoma using monoclonal antibody MIB1. A comparison with proliferating cell nuclear antigen. Am J Clin Pathol 104:313-318. DOI 10.1093/ajcp/104.3.313.

IR K, O F, G H, E vM, and MV K. 2017. What is precision medicine? Eur Respir J 50: 1700391. DOI 10.1183/13993003.00391-2017.

Isohookana J, Haapasaari KM, Soini Y, Leppanen J, and Karihtala P. 2018. Proteins of the retinoblastoma pathway, FEN1 and MGMT are novel potential prognostic biomarkers in pancreatic adenocarcinoma. Pathol Res Pract 214:840-847. DOI 10.1016/j.prp.2018.04.016.

J Z, and CB T. 2019. Metabolic regulation of cell growth and proliferation. Nat Rev Mol Cell Biol 20:436-450. DOI 10.1038/s41580-019-0123-5.

JC H, WW S, HP T, KC H, CM Y, CJ C, HC T, CT H, GS S, JG C, KT Y, and TC L. 2015. The Overexpression of FEN1 and RAD54B May Act as Independent Prognostic Factors of Lung Adenocarcinoma. PLoS One 10:e0139435. DOI 10.1371/journal.pone.0139435.

K S, M K, S T, T M, T M, and K S. 1995. A long-term survivor of ruptured hepatocellular carcinoma after hepatic resection. J Gastroenterol Hepatol 10:351-354.

$\mathrm{K} \mathrm{W}, \mathrm{C} \mathrm{X}$, and D C. 2014. Flap endonuclease 1 is a promising candidate biomarker in gastric cancer and is involved in cell proliferation and apoptosis. Int J Mol Med 33:1268-1274. DOI 10.3892/ijmm.2014.1682.

Kuo TC, Chang PY, Huang SF, Chou CK, and Chao CC. 2012. Knockdown of HURP inhibits the proliferation of hepacellular carcinoma cells via downregulation of gankyrin and accumulation of p53. Biochem Pharmacol 83:758-768. DOI 10.1016/j.bcp.2011.12.034.

L H, L L, H Z, H Y, Y Z, H W, H S, F J, CS K, L L, Z Z, H C, F P, Z H, J Z, and Z G. 2017. FEN1 promotes tumor progression and confers cisplatin resistance in non-small-cell lung cancer. Mol Oncol 11:640-654. DOI 10.1002/1878-0261.12058.

L Z, J J, LD F, Z G, C Z, and B S. 2011. Functional regulation of FEN1 nuclease and its link to cancer. Nucleic Acids Res 39:781-794. DOI 10.1093/nar/gkq884.

Lam JS, Seligson DB, Yu H, Li A, Eeva M, Pantuck AJ, Zeng G, Horvath S, and Belldegrun AS. 2006. Flap endonuclease 1 is overexpressed in prostate cancer and is associated with a

Peer] reviewing PDF | (2019:06:38406:1:1:NEW 1 Aug 2019) 
569

570

571

572

573

574

575

576

577

578

579

580

581

582

583

584

585

586

587

588

589

590

591

592

593

594

595

596

597

598

599

600

601

602

603

604

605

606

607

608

609

610

611

612

613

614

high Gleason score. BJU Int 98:445-451. DOI 10.1111/j.1464-410X.2006.06224.x.

Liao W, Liu W, Yuan Q, Liu X, Ou Y, He S, Yuan S, Qin L, Chen Q, Nong K, Mei M, and Huang J. 2013. Silencing of DLGAP5 by siRNA significantly inhibits the proliferation and invasion of hepatocellular carcinoma cells. PLoS One 8:e80789. DOI 10.1371/journal.pone.0080789.

Lieber MR. 1997. The FEN-1 family of structure-specific nucleases in eukaryotic DNA replication, recombination and repair. Bioessays 19:233-240. DOI 10.1002/bies.950190309.

Liu L, Chen X, Xie S, Zhang C, Qiu Z, and Zhu F. 2012a. Variant 1 of KIAA0101, overexpressed in hepatocellular carcinoma, prevents doxorubicin-induced apoptosis by inhibiting p53 activation. Hepatology 56:1760-1769. DOI 10.1002/hep.25834.

Liu L, Zhou C, Zhou L, Peng L, Li D, Zhang X, Zhou M, Kuang P, Yuan Q, Song X, and Yang M. 2012b. Functional FEN1 genetic variants contribute to risk of hepatocellular carcinoma, esophageal cancer, gastric cancer and colorectal cancer. Carcinogenesis 33:119-123. DOI 10.1093/carcin/bgr250.

Liu Z, Li J, Chen J, Shan Q, Dai H, Xie H, Zhou L, Xu X, and Zheng S. 2018. MCM family in HCC: MCM6 indicates adverse tumor features and poor outcomes and promotes S/G2 cell cycle progression. BMC Cancer 18:200. DOI 10.1186/s12885-018-4056-8.

M A, N K, N I, A H, K H, O M, and M Y. 2009. The knockdown of endogenous replication factor $\mathrm{C} 4$ decreases the growth and enhances the chemosensitivity of hepatocellular carcinoma cells. Liver Int 29:55-62. DOI 10.1111/j.1478-3231.2008.01792.x.

M L, and J Z-R. 2016. Mechanisms of HBV-induced hepatocellular carcinoma. J Hepatol 64:S84-S101. DOI 10.1016/j.jhep.2016.02.021.

MJ D, N OD, DJ B, WM G, and BM R. 2007. Survivin: a promising tumor biomarker. Cancer Lett 249:49-60. DOI 10.1016/j.canlet.2006.12.020.

MP K, Y Z, and G L. 2015. Mutant p53: Multiple Mechanisms Define Biologic Activity in Cancer. Front Oncol 5:249. DOI 10.3389/fonc.2015.00249.

Nikolova T, Christmann M, and Kaina B. 2009. FEN1 is overexpressed in testis, lung and brain tumors. Anticancer Res 29:2453-2459.

P H, Y W, A F, X L, V M, T O, YJ C, KE S, JM S, H Y, RL B, S G, H Y, KJ K, J Y, C J, Y L, D F, R S, K N, E S, L A, JJ Z, K P, DG A, C L, and P S. 2017. Cell-Cycle-Targeting MicroRNAs as Therapeutic Tools against Refractory Cancers. Cancer cell 31:576590.e578. DOI 10.1016/j.ccell.2017.03.004.

Pan HW, Chou HY, Liu SH, Peng SY, Liu CL, and Hsu HC. 2006. Role of L2DTL, cell cycleregulated nuclear and centrosome protein, in aggressive hepatocellular carcinoma. Cell Cycle 5:2676-2687. DOI 10.4161/cc.5.22.3500.

PN R. 2012. Deep phenotyping for precision medicine. Hum Mutat 33:777-780. DOI 10.1002/humu.22080.

R N, H J, CJ T, and F C. 2019. Review: Precision medicine and driver mutations: Computational methods, functional assays and conformational principles for interpreting cancer drivers. PLoS Comput Biol 15:e1006658. DOI 10.1371/journal.pcbi.1006658.

S R, EL L, A B, Y C, X Z, J J, R W, HL J, QH Y, LX Q, ZY T, P H, KW H, SS T, PS M, and $\mathrm{XW}$ W. 2012. Integrative genomic identification of genes on $8 \mathrm{p}$ associated with hepatocellular carcinoma progression and patient survival. Gastroenterology 142:957966.e912. DOI 10.1053/j.gastro.2011.12.039.

S R, HL J, A B, M F, QH Y, JS L, SS T, Z S, ZY T, LX Q, and XW W. 2010. A unique

Peer) reviewing PDF | (2019:06:38406:1:1:NEW 1 Aug 2019) 
615

616

617

618

619

620

621

622

623

624

625

626

627

628

629

630

631

632

633

634

635

636

637

638

639

640

641

642

643

644

645

646

647

648

649

650

651

652

653

654

655

656

657

658

659

660

metastasis gene signature enables prediction of tumor relapse in early-stage hepatocellular carcinoma patients. Cancer Res 70:10202-10212. DOI 10.1158/00085472.CAN-10-2607.

Sang L, Wang XM, Xu DY, and Zhao WJ. 2018. Bioinformatics analysis of aberrantly methylated-differentially expressed genes and pathways in hepatocellular carcinoma. World J Gastroenterol 24:2605-2616. DOI 10.3748/wjg.v24.i24.2605.

Shen S, Kong J, Qiu Y, Yang X, Wang W, and Yan L. 2019. Identification of core genes and outcomes in hepatocellular carcinoma by bioinformatics analysis. $J$ Cell Biochem 120:10069-10081. DOI 10.1002/jcb.28290.

T M, K T, E A, T M, K S, H H, K S, and M T. 1995. Small hepatocellular carcinoma of single nodular type: a specific reference to its surrounding cancerous area undetected radiologically and macroscopically. J Surg Oncol 60:75-79.

T S, Y I, K T, T S, T M, K T, Y H, M N, T I, T F, M O, K E, T U, Y N, S O, M O, T K, R I, Y T, R O, D Y, K T, T F, J P, MS L, T U, T O, T M, T W, H K, T O, T N, H M, S W, T S, M $\mathrm{Y}, \mathrm{K} \mathrm{T}$, and M K. 2016. p62/Sqstm1 promotes malignancy of HCV-positive hepatocellular carcinoma through Nrf2-dependent metabolic reprogramming. Nat Commun 7:12030. DOI 10.1038/ncomms12030.

Tsou AP, Yang CW, Huang CY, Yu RC, Lee YC, Chang CW, Chen BR, Chung YF, Fann MJ, Chi CW, Chiu JH, and Chou CK. 2003. Identification of a novel cell cycle regulated gene, HURP, overexpressed in human hepatocellular carcinoma. Oncogene 22:298-307. DOI 10.1038/sj.onc.1206129.

W C, W D, J W, Z W, and X H. 2019. Elevated Expressions of Survivin and Endoglin in Patients with Hepatic Carcinoma. Cancer Biother Radiopharm 34:7-12. DOI 10.1089/cbr.2018.2539.

Wang K, Xie C, and Chen D. 2014. Flap endonuclease 1 is a promising candidate biomarker in gastric cancer and is involved in cell proliferation and apoptosis. Int J Mol Med 33:12681274. DOI 10.3892/ijmm.2014.1682.

WG Z, CF L, M L, XF C, K S, X K, L L, and ZC M. 2016. Aquaporin 9 is down-regulated in hepatocellular carcinoma and its over-expression suppresses hepatoma cell invasion through inhibiting epithelial-to-mesenchymal transition. Cancer Lett. 378:111-119. DOI 10.1016/j.canlet.2016.05.021.

Wu X, Wilson TE, and Lieber MR. 1999. A role for FEN-1 in nonhomologous DNA end joining: the order of strand annealing and nucleolytic processing events. Proc Natl Acad Sci US A 96:1303-1308. DOI 10.1073/pnas.96.4.1303.

X Z, X Q, C Z, L X, K H, Y L, N Z, J F, S S, L Z, J X, Z G, Y T, and X C. 2019. FEN1 mediates miR-200a methylation and promotes breast cancer cell growth MET and EGFR signaling. FASEB J 2:fj201900273R. DOI 10.1096/fj.201900273R.

Y L, F R, Y L, Z S, Z T, H X, Y D, and G C. 2015. Clinicopathological and prognostic significance of high Ki-67 labeling index in hepatocellular carcinoma patients: a metaanalysis. Int J Clin Exp Med 8:10235-10247.

YH W, TY C, TY C, KM C, VP C, and KJ K. 2014. Plasmalemmal Vesicle Associated Protein (PLVAP) as a therapeutic target for treatment of hepatocellular carcinoma. BMC Cancer 14:815. DOI 10.1186/1471-2407-14-815.

Yuan RH, Jeng YM, Pan HW, Hu FC, Lai PL, Lee PH, and Hsu HC. 2007. Overexpression of KIAA0101 predicts high stage, early tumor recurrence, and poor prognosis of hepatocellular carcinoma. Clin Cancer Res 13:5368-5376. DOI 10.1158/1078-0432.ccr- 
$661 \quad 07-1113$.

662 Zhang K, Keymeulen S, Nelson R, Tong TR, Yuan YC, Yun X, Liu Z, Lopez J, Raz DJ, and

663 Kim JY. 2018. Overexpression of Flap Endonuclease 1 Correlates with Enhanced

664 Proliferation and Poor Prognosis of Non-Small-Cell Lung Cancer. Am J Pathol 188:242-

665 251. DOI 10.1016/j.ajpath.2017.09.011. 
Figure 1

Venn diagram.

(A) 409 DEGs were identified in 4 datasets (GSE14520, GSE29721, GSE45267, and GSE60502) via FUNRICH. These included 142 upregulated genes. (B) and 267 downregulated genes. (C) Colors correspond to specific datasets, with intersecting areas indicating overlapping gene sets. DEG identification criteria were adj. $\mathrm{P}<0.05$ and |log FC (fold change) $\mid \geq 1$.
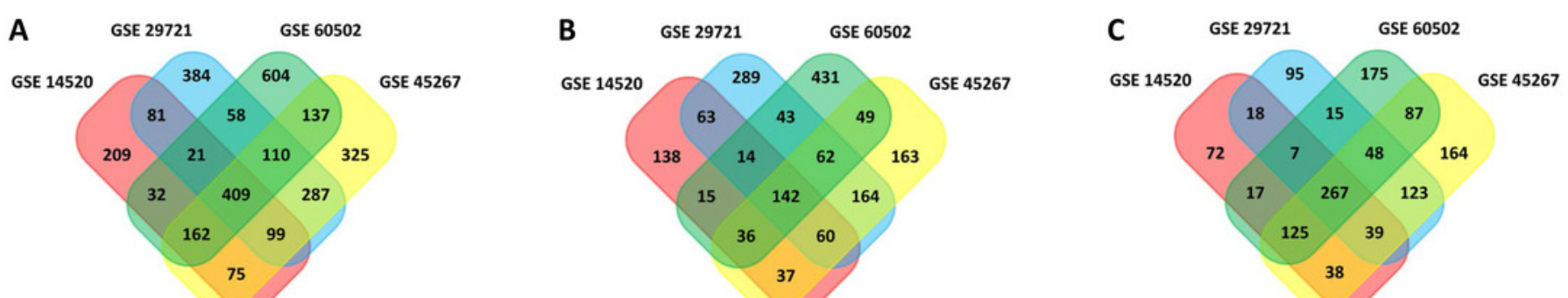


\section{Figure 2}

DEG PPI network and modular analysis.

(A) STRING was employed to create a PPI network of 403 nodes and 3502 edges, visualized using Cytoscape software. Genes that are upregulated are shown by red nodes, while those that are downregulated are blue. The MCODE plug-in was used to analyze highlighted regions. (B) Clustering module 1 scored 58.492 with 62 nodes and 1784 edges. (C) Clustering module 2 scored 11.529 with 18 nodes and 98 edges. (D) Clustering module 3 scored 10.917 with 25 nodes and 131 edges.
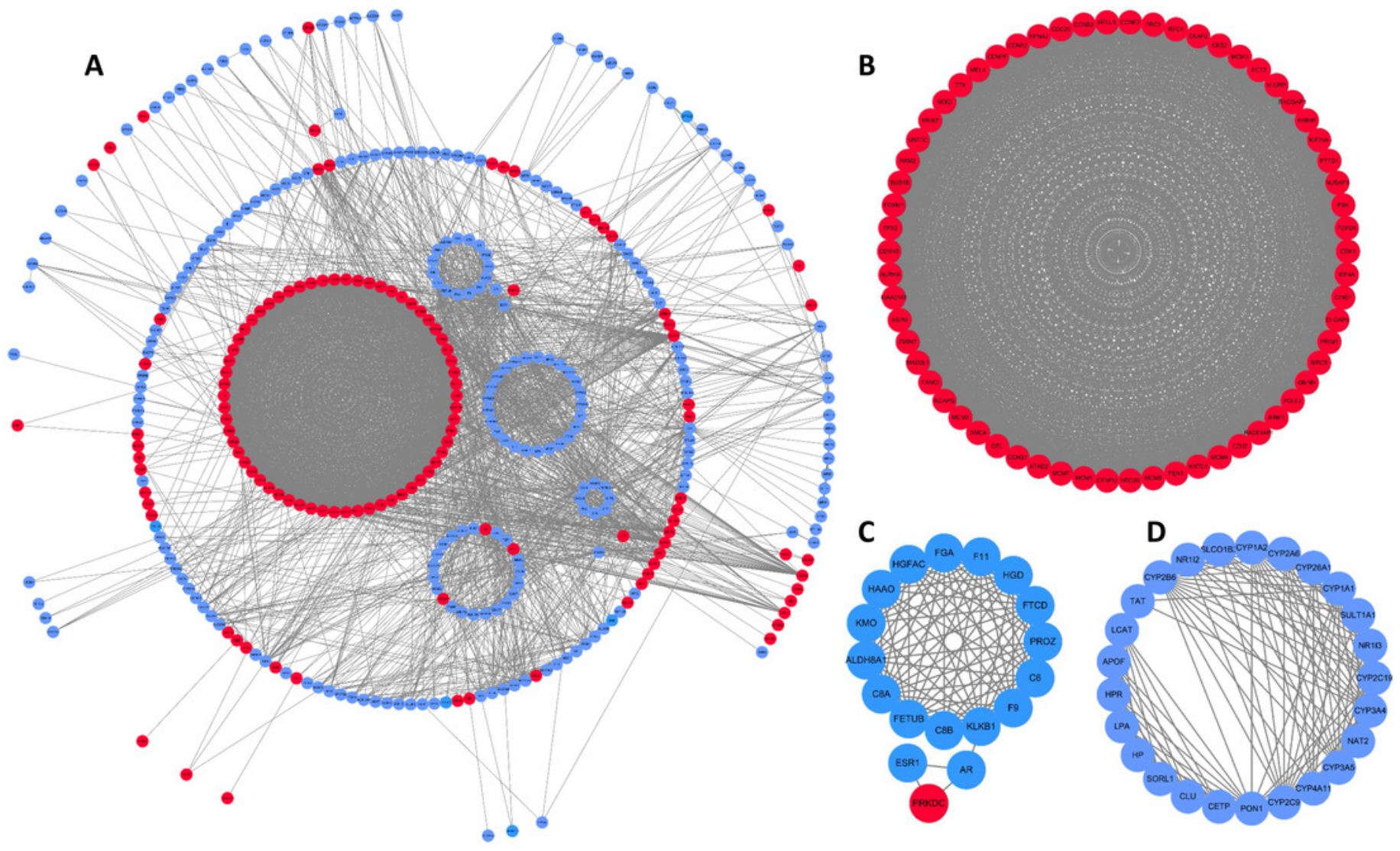


\section{Figure 3}

Identification of nine core HCC-associated genes.

(A) Nine core genes in clustering module 1 screened by 12 algorithms using the cytoHubba plug-in intersected with Hub genes. (B) Core gene expression profiles in 421 TCGA liver cancer tissues, including 50 solid normal tissues and 371 primary tumors, **P $<0.01$. (C) The UCSC databased was used for core gene hierarchical clustering.
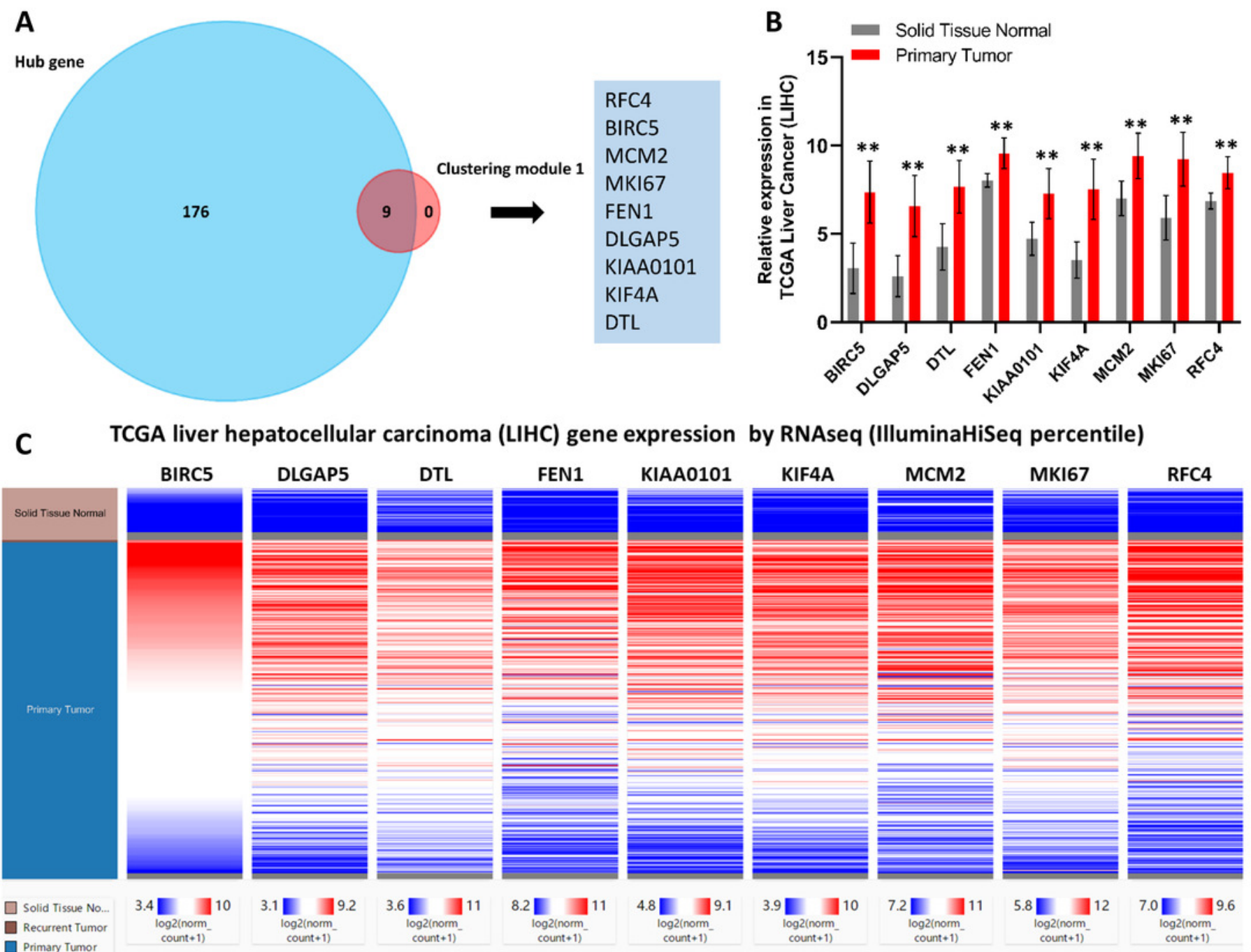
Figure 4

Association between core genes and overall survival.

Association between core genes and overall survival (A, BIRC5; B, DLGAP5; C, DTL; D, FEN1;

E, KIAA0101; F, KIF4A; G, MCM2; H, MKI67 and I, RFC4) in those with HCC. Cl, confidence interval; HR, hazard ratio. High- and low-risk groups are shown in red and blue, respectively. $P<0.05$ was the significance threshold. 

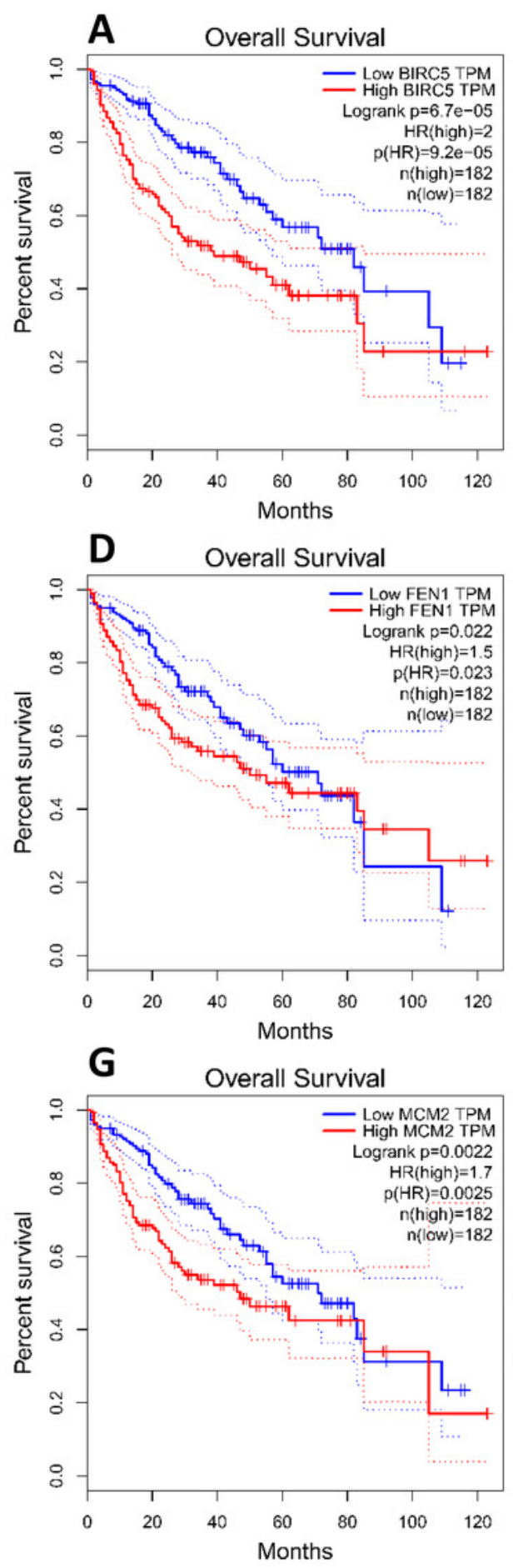

B
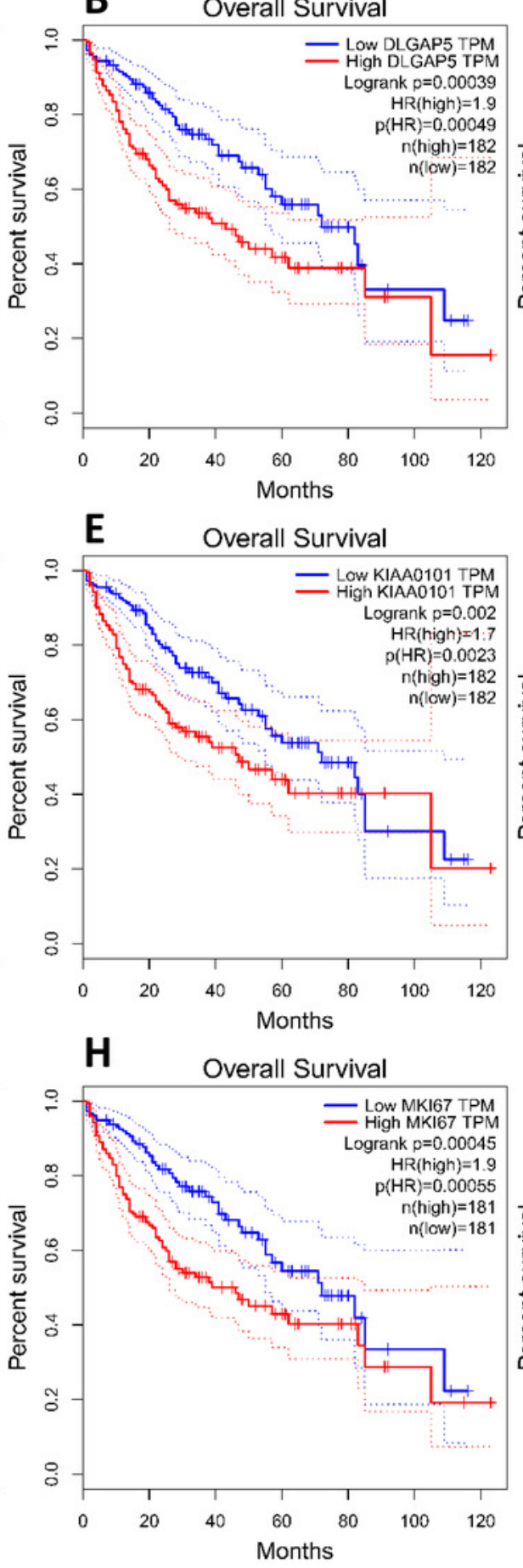
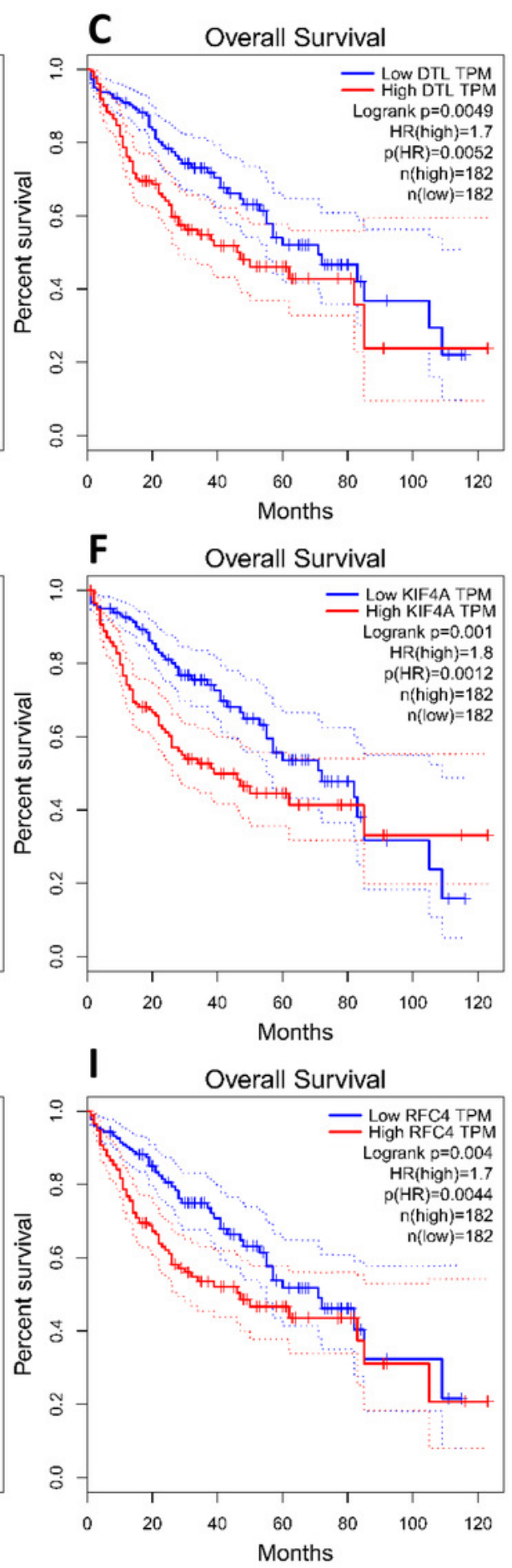


\section{Figure 5}

Association between core genes and disease-free survival.

Association between core genes and disease-free survival (A, BIRC5; B, DLGAP5; C, DTL; D, FEN1; E, KIAA0101; F, KIF4A; G, MCM2; H, MKI67 and I, RFC4) in those with HCC. Cl, confidence interval; HR, hazard ratio. High- and low-risk groups are shown in red and blue, respectively. $\mathrm{P}<0.05$ was the significance threshold. 

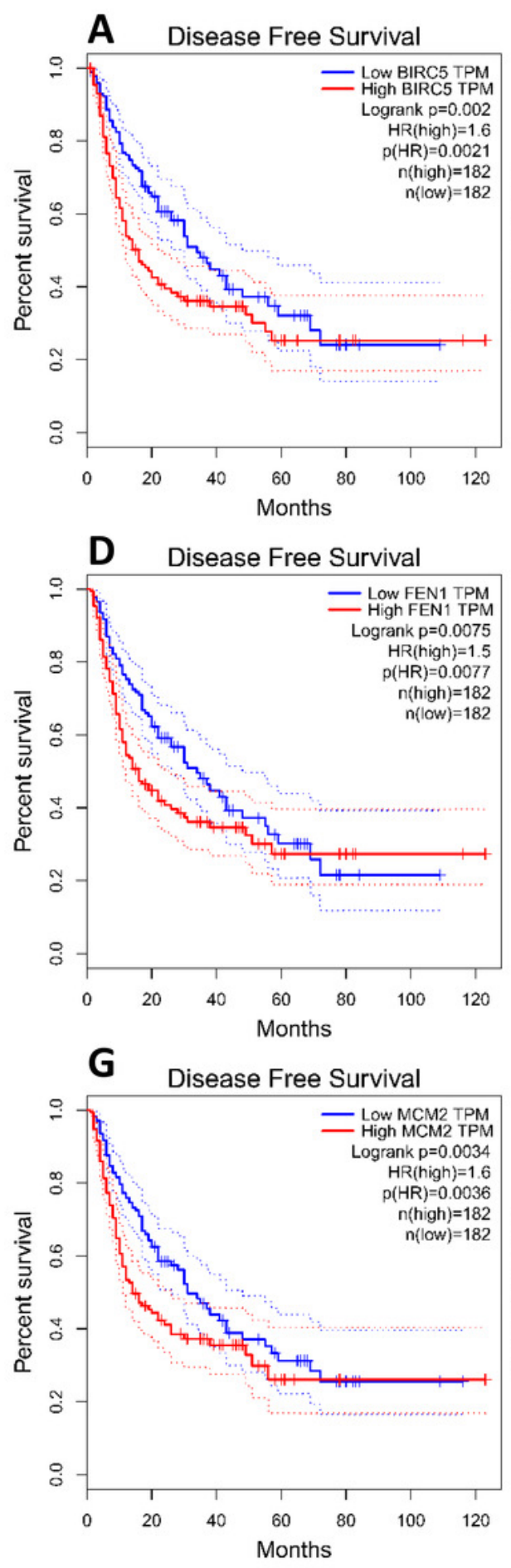

B Disease Free Survival
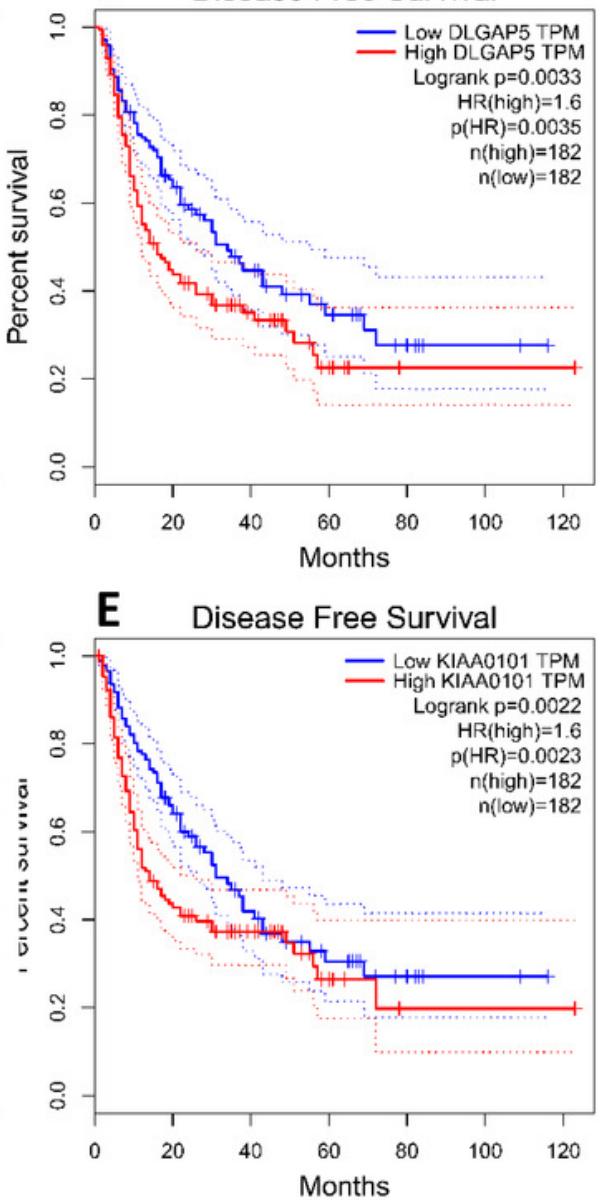

\section{H Disease Free Survival}

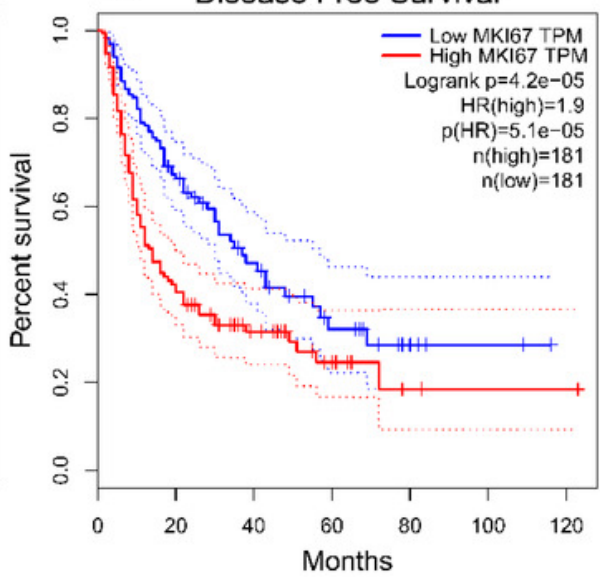

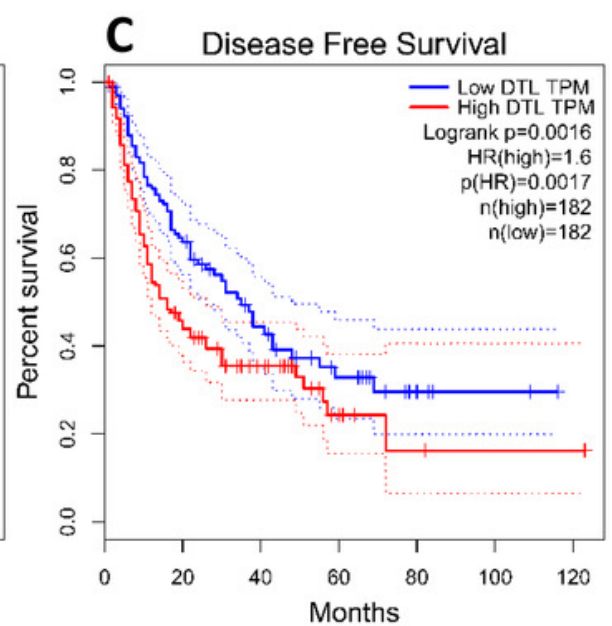
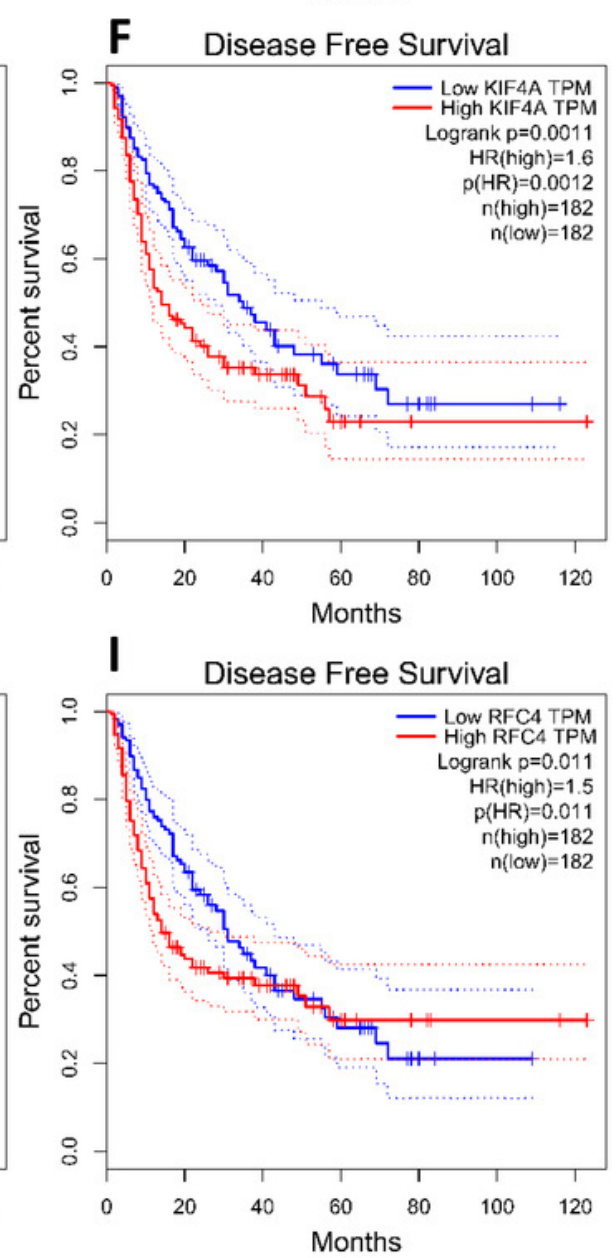


\section{Figure 6}

Correlations between the expression of FEN1 and MCM2, BIRC5, and RFC4.

(A) A PPI network for the nine core genes generated with the Cbioportal database; (B) The correlation between FEN1 and MCM2 in 421 TCGA liver cancer tissues including 50 solid normal tissues and 371 primary tumors; (C) The correlation between FEN1 and BIRC5 in 421 TCGA liver cancer tissues including 50 solid normal tissues and 371 primary tumors; (D) The correlation between FEN1 and RFC4 in 421 TCGA liver cancer tissues including 50 solid normal tissues and 371 primary tumors. $\mathrm{P}<0.05$ was the significance threshold. 


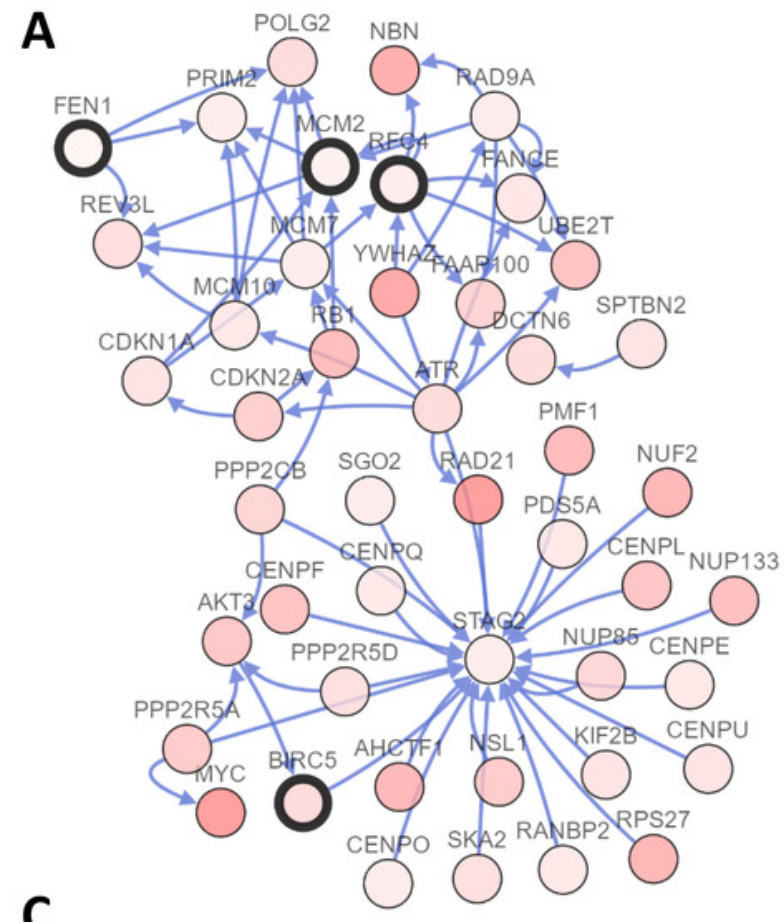

B
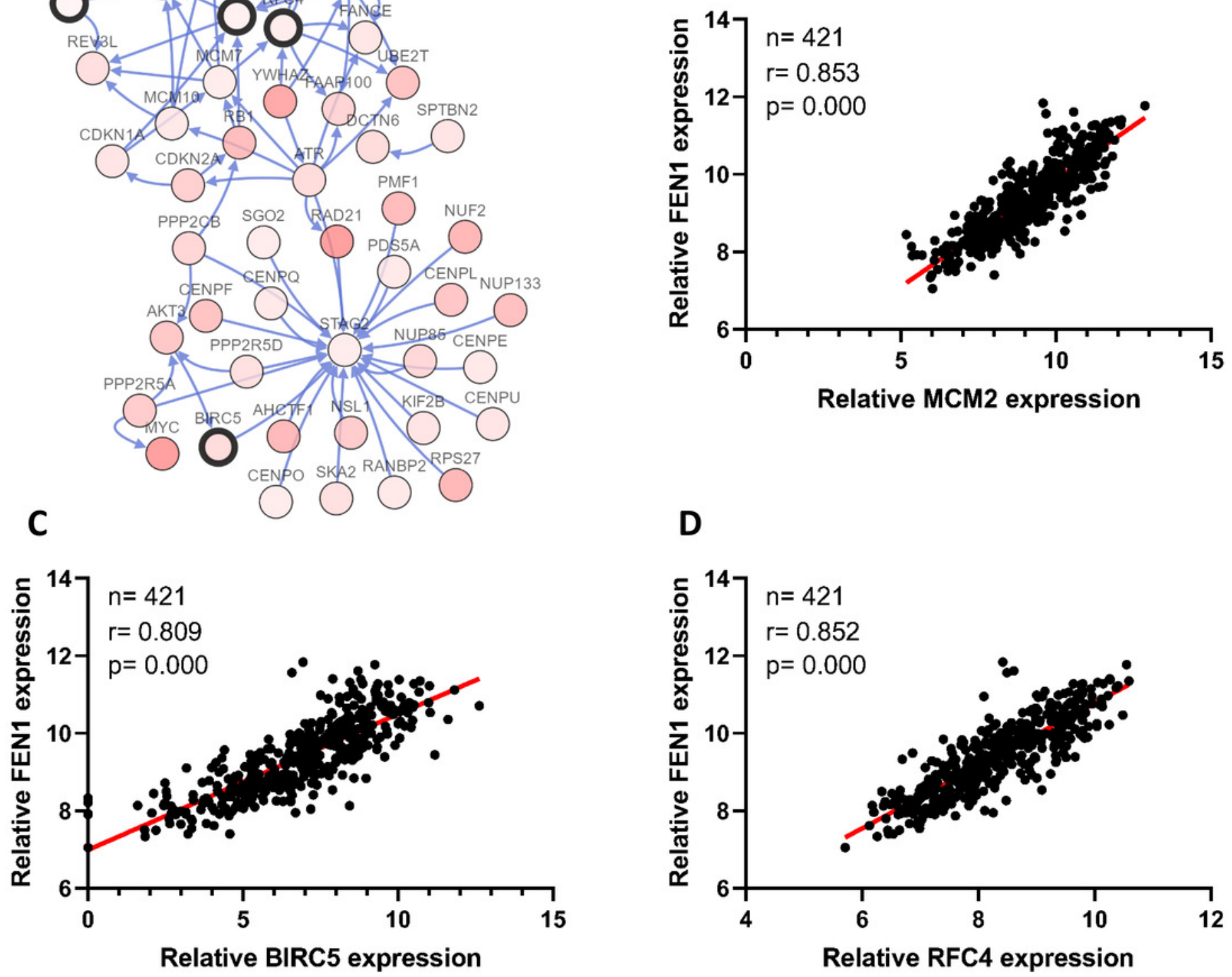
Figure 7

The expression of FEN1 in cancers including HCC.

The expression of FEN1 in various tumor tissue types was analyzed in the ONCOMINE (A) and

FIREBROWSE databases (B); (C) The expression of FEN1 in three different HCC-related chip data sets was analyzed in the ONCOMINE database.

A Disease Summary for FEN1

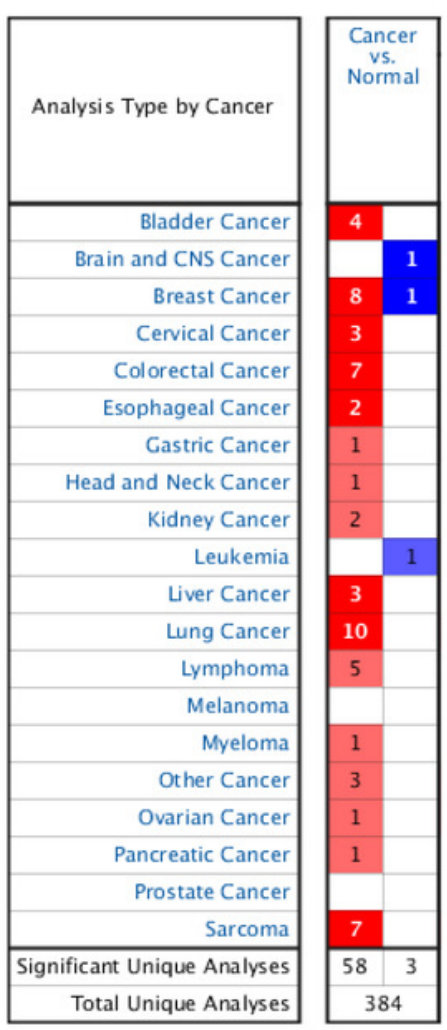

$\stackrel{5}{5} \square^{10} \square \square^{10 \quad 5} \square^{1}$

Cell color is determined by the best gene rank percentile for the analyses within the cell.

NOTE: An analysis may be counted in more than one cancer type.

B

C

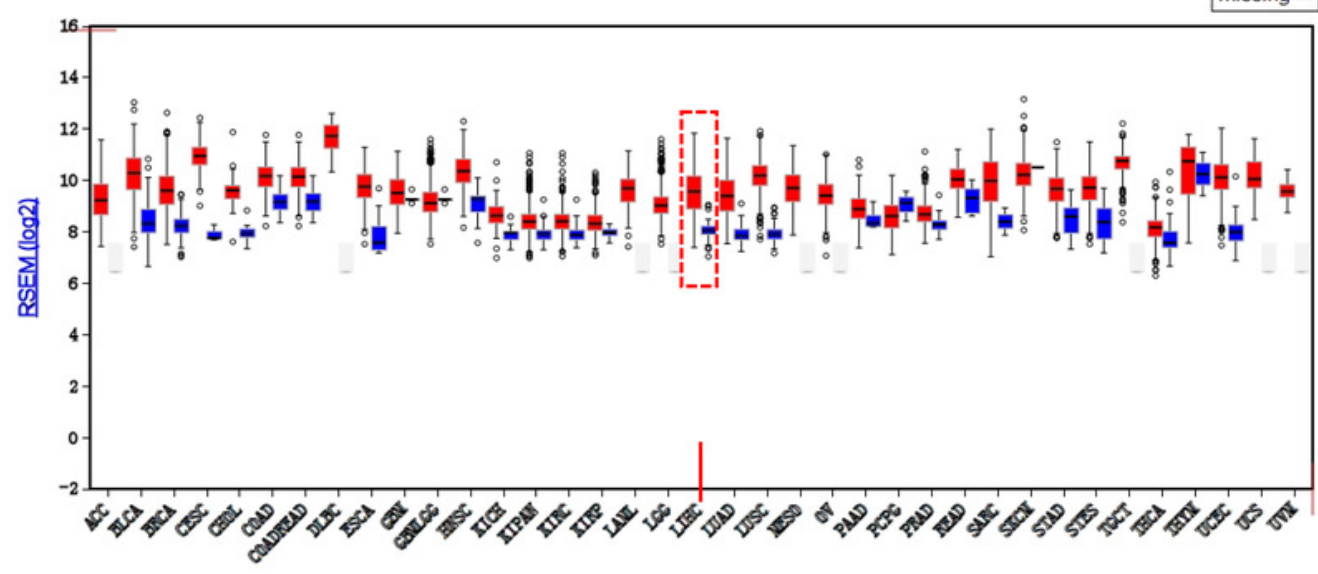

Comparison of FEN1 Across 3 Analyses

Over-expression

Median Rank p-Value Gene

$108.0 \quad 2.02 \mathrm{E}-18 \quad$ FEN 1

\begin{tabular}{|l|l|l|}
\hline 1 & 2 & 3 \\
\hline
\end{tabular}

Legend

1. Hepatocellular Carcinoma vs. Normal 3. Hepatocellular Carcinoma vs. Normal Chen Liver, Mol Biol Cell, 2002 Roessler Liver 2, Cancer Res, 2010

2. Hepatocellular Carcinoma vs. Normal

Roessler Liver, Cancer Res, 2010

$\begin{array}{llllllll}1 & 5 & 10 & 25 & 25 & 10 & 5 & 1\end{array}$

$\stackrel{\square}{\longleftarrow} \square \square \square \square \square \square \square$ Not measured

The rank for a gene is the median rank for that gene across each of

The $\mathrm{p}$-Value for a gene is its p-Value for the median-ranked analysis. 


\section{Figure 8}

FEN1 was up-regulated in HCC tissues and hepatoma cell lines.

H\&E staining of adjacent tissue (A) and HCC tissue (C). (B) FEN1 IHC in adjacent tissue. (D) FEN1 IHC in HCC tissue. (E) FEN1 IHC staining quantification; $n=34 /$ group. (F) FEN1 IHC scores; $n=34$ /group. (G) FEN1 expression in control HL7702 liver cells and in hepatoma cell lines (as indicated); $* \mathrm{P}<0.05, * * \mathrm{P}<0.01$. 


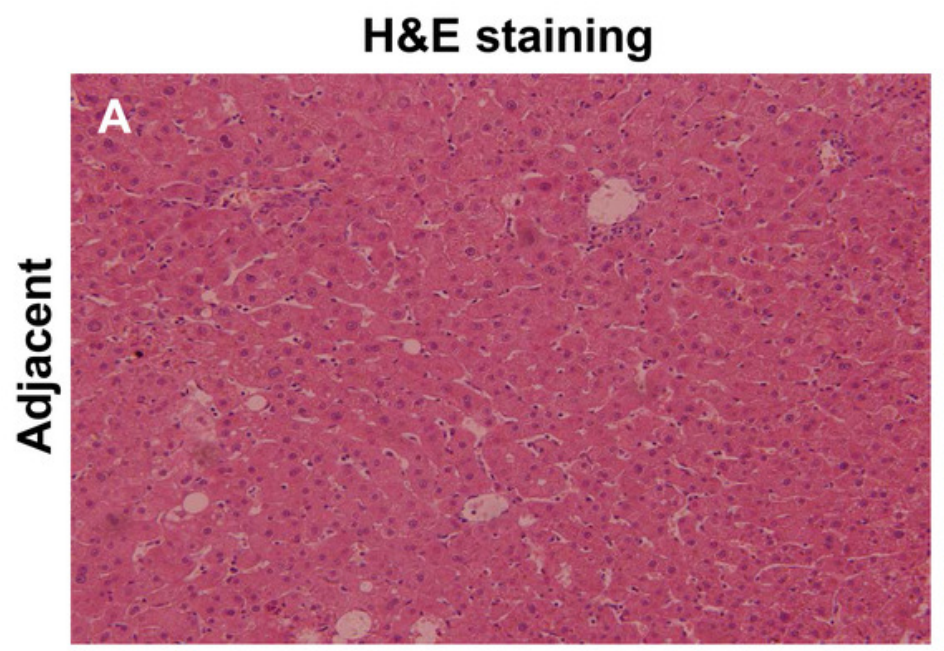

FEN1 IHC
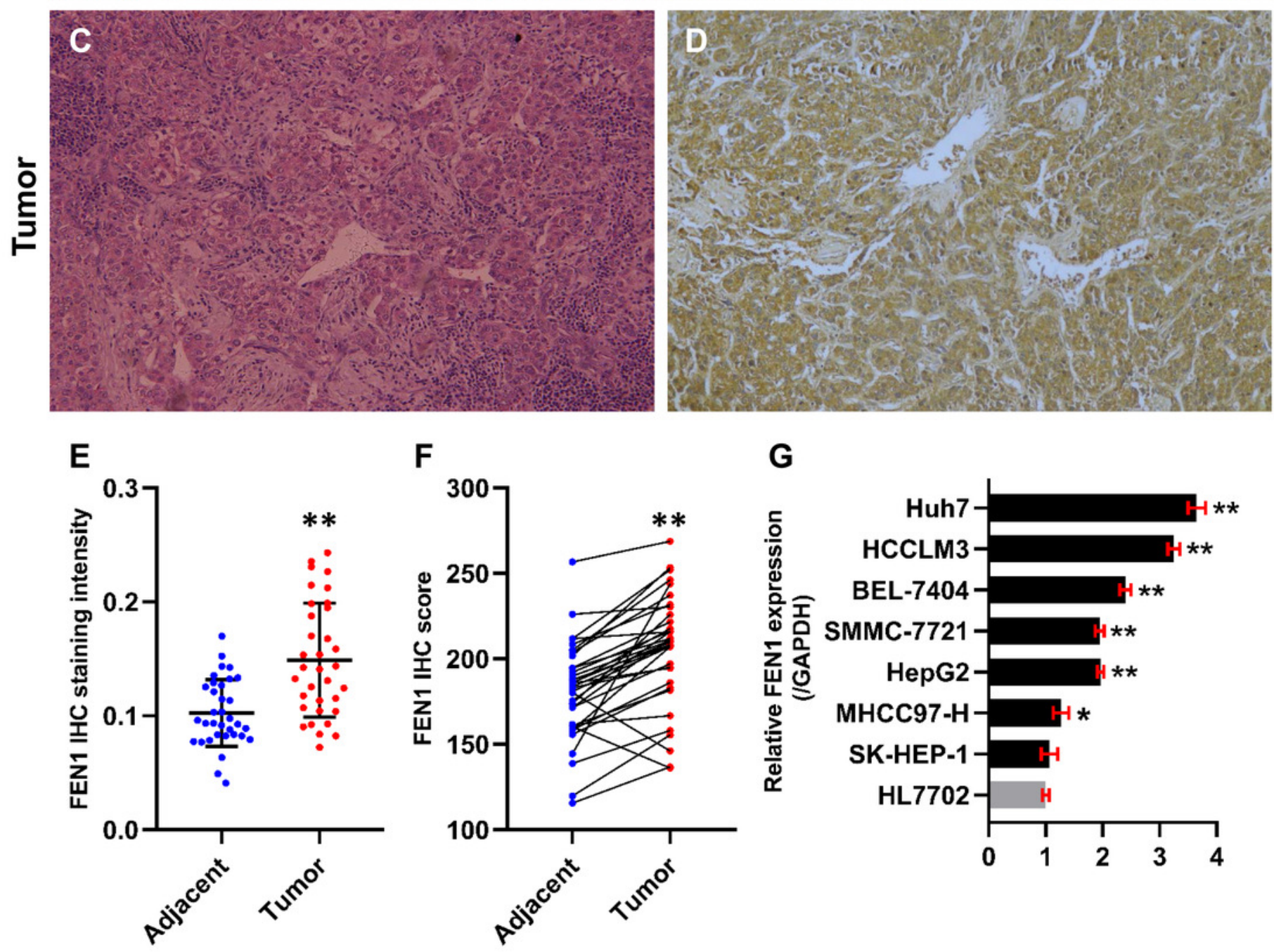
Table $\mathbf{1}$ (on next page)

Detailed information of the GEO datasets in this study. 
Table.1 Detailed information of the GEO datasets in this study.

\begin{tabular}{|c|c|c|c|c|c|}
\hline $\begin{array}{c}\text { Series } \\
\text { accession }\end{array}$ & Species & & ype & & Platform \\
\hline GSE14520 & Homo sapiens & $\begin{array}{l}\text { Expression } \\
\text { array }\end{array}$ & profiling & by & $\begin{array}{l}\text { GPL3921 Affymetrix HT Human } \\
\text { Genome U133A Array }\end{array}$ \\
\hline GSE29721 & Homo sapiens & $\begin{array}{l}\text { Expression } \\
\text { array }\end{array}$ & profiling & by & $\begin{array}{l}\text { GPL570 [HG-U133_Plus_2] } \\
\text { Affymetrix Human Genome U133 } \\
\text { Plus } 2.0 \text { Array }\end{array}$ \\
\hline GSE45267 & Homo sapiens & $\begin{array}{l}\text { Expression } \\
\text { array }\end{array}$ & profiling & by & $\begin{array}{l}\text { GPL570 [HG-U133_Plus_2] } \\
\text { Affymetrix Human Genome U133 } \\
\text { Plus } 2.0 \text { Array }\end{array}$ \\
\hline GSE60502 & Homo sapiens & $\begin{array}{l}\text { Expression } \\
\text { array }\end{array}$ & profiling & by & $\begin{array}{l}\text { GPL96 [HG-U133A] Affymetrix } \\
\text { Human Genome U133A Array }\end{array}$ \\
\hline
\end{tabular}

2 GEO, Gene Expression Omnibus.

3 
Table 2 (on next page)

Correlation between FEN1 expression and clinicopathological features in 34 paired HCC patients 
1

2

3

Table 2 Correlation between FEN1 expression and clinicopathological features in 34 paired HCC patients

\begin{tabular}{|c|c|c|c|c|}
\hline \multirow{2}{*}{$\begin{array}{c}\text { Clinicopathological } \\
\text { features }\end{array}$} & \multirow{2}{*}{$\begin{array}{l}\text { Cases } \\
(n=34)\end{array}$} & \multicolumn{2}{|c|}{ FEN1 expression } & \multirow[t]{2}{*}{$P$ value } \\
\hline & & High (\%) & Low (\%) & \\
\hline All case $(n=34)$ & 34 & 25 & 9 & \\
\hline Gender & & & & 1.000 \\
\hline Male & 29 & $21(61.8 \%)$ & $8(23.5 \%)$ & \\
\hline Female & 5 & $4(11.8 \%)$ & $1(2.9 \%)$ & \\
\hline $\operatorname{Age}(y)^{a}$ & & & & 0.697 \\
\hline$<52$ & 15 & $12(35.3 \%)$ & $3(8.8 \%)$ & \\
\hline$\geq 52$ & 19 & $13(38.2 \%)$ & $6(17.6 \%)$ & \\
\hline Tumor size & & & & $0.047^{*}$ \\
\hline$<5 \mathrm{~cm}$ & 19 & $11(32.4 \%)$ & $8(23.5 \%)$ & \\
\hline$\geq 5 \mathrm{~cm}$ & 15 & $14(41.2 \%)$ & $1(2.9 \%)$ & \\
\hline Tumor multiplicity & & & & 0.348 \\
\hline Single & 27 & $21(61.8 \%)$ & $6(17.6 \%)$ & \\
\hline Multiple & 7 & $4(11.8 \%)$ & $3(8.8 \%)$ & \\
\hline TNM stage & & & & 1.000 \\
\hline $\mid \sim \|$ & 23 & $17(50.0 \%)$ & $6(17.6 \%)$ & \\
\hline III IV & 11 & $8(23.5 \%)$ & $3(8.8 \%)$ & \\
\hline Pathological grade & & & & 1.000 \\
\hline Well/moderate & 27 & $20(58.8 \%)$ & $7(20.6 \%)$ & \\
\hline Poor & 7 & $5(14.7 \%)$ & $2(5.9 \%)$ & \\
\hline Metastasis & & & & $0.013^{*}$ \\
\hline With & 12 & $12(35.3 \%)$ & $0(0.0 \%)$ & \\
\hline Without & 22 & $13(38.2 \%)$ & $9(26.5 \%)$ & \\
\hline HBsAg & & & & 1.000 \\
\hline Positive & 30 & $22(64.7 \%)$ & $8(23.5 \%)$ & \\
\hline Negative & 4 & $3(8.8 \%)$ & $1(2.9 \%)$ & \\
\hline Liver cirrhosis & & & & 0.687 \\
\hline With & 12 & $8(23.5 \%)$ & $4(11.8 \%)$ & \\
\hline Without & 22 & $17(50.0 \%)$ & $5(14.7 \%)$ & \\
\hline Serum AFP & & & & 0.725 \\
\hline$>400 \mathrm{ng} / \mathrm{ml}$ & 18 & $12(35.3 \%)$ & $6(17.6 \%)$ & \\
\hline$\leqslant 400 \mathrm{ng} / \mathrm{ml}$ & 16 & $9(26.5 \%)$ & $7(20.6 \%)$ & \\
\hline Vascular invasion & & & & $0.024^{*}$ \\
\hline Present & 14 & $13(38.2 \%)$ & $1(2.9 \%)$ & \\
\hline Absent & 20 & 11(32.4\%) & $9(26.5 \%)$ & \\
\hline
\end{tabular}

4 a: patients were divided according to the median age; AFP: alpha-fetoprotein; HBsAg: hepatitis B surface 5 antigen; $*: P<0.05$. 